\title{
Reaction time distribution analysis of spatial correspondence effects
}

\author{
Robert W. Proctor • James D. Miles • Giulia Baroni
}

Published online: 12 February 2011

(C) Psychonomic Society, Inc. 2011

\begin{abstract}
Since 1994, group reaction time (RT) distribution analyses of spatial correspondence effects have been used to evaluate the dynamics of the spatial Simon effect, a benefit of correspondence of stimulus location information with response location for tasks in which stimulus location is irrelevant. We review the history and justification for analyzing group RT distributions and clarify which conditions result in the Simon effect decreasing across the distribution and which lead to flat or increasing functions. Although the standard left-right Simon effect typically yields a function for which the effect decreases as RT increases, in most other task variations, the Simon effect remains stable or increases across the RT distribution. Studies that have used other means of evaluating the temporal dynamics of the Simon effect provide converging evidence that the changes in the Simon effect across the distribution are due mainly to temporal activation properties, an issue that has been a matter of some dispute.
\end{abstract}

Keywords Correspondence effects · Delta plots · Simon effect - Stimulus-response compatibility - Time course of processing

In the late 1960s and early 1970s, J. R. Simon reported the results of reaction time (RT) studies in which participants

R. W. Proctor $(\bowtie) \cdot J$. D. Miles

Department of Psychological Sciences, Purdue University, 703 Third St.,

W. Lafayette, IN 47907-2081, USA

e-mail: proctor@psych.purdue.edu

G. Baroni

Communication Disciplines Department, University of Bologna,

Via A. Gardino, 23 - 40122,

Bologna, Italy made a left or right manual response to a feature of a stimulus (e.g., red-green color of a visual stimulus) presented in a left or a right location (see Fig. 1; see, e.g., Simon \& Small, 1969; see Simon, 1990, for a review). RT was shorter when stimulus and response locations corresponded than when they did not, a result called the Simon effect (Hedge \& Marsh, 1975). This two-choice task has come to be prototypical, and we refer to it and the resulting Simon effect as standard. The term Simon effect has been extended to include correspondence effects for other irrelevant stimulus dimensions that overlap with, or are similar to, a response dimension (Kornblum, Hasbroucq, \& Osman, 1990), but we focus on task variations that entail correspondence of spatial information and manual responses.

Studies of the Simon effect and related correspondence effects focused on differences in mean RT at first (see Lu \& Proctor, 1995), and these differences are still the focal point of a lot of investigations. However, beginning with De Jong, Liang, and Lauber (1994), many authors have also reported analyses of the RT distributions, dividing them into quantiles, or bins, that contain RTs in the range of percentile values (e.g., between the 50th and 60th percentiles) and measuring the Simon effect for each bin. De Jong et al. (1994) found that the Simon effect for visual tasks was largest at the short RT bins and decreased across the distribution. This result has typically been attributed to rapid activation of the response code corresponding to the irrelevant location feature, followed by decrease of that activation. But, this temporal activation account has been debated (e.g., Roswarski \& Proctor, 2003; Zhang \& Kornblum, 1997), and the pattern of decreasing effect size across the RT distribution has not been found in several task variations.

Many studies now exist for which RT distribution analyses have been reported, and it has become routine to include them in articles (e.g., Davranche \& McMorris, 

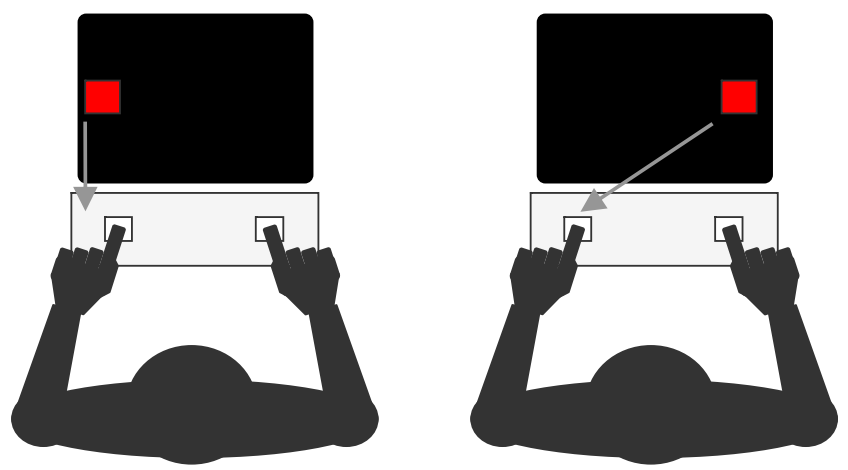

Fig. 1 Example of the standard Simon task. One stimulus occurs in a left or right location, but stimulus location is irrelevant to the task: Participants are to press a left/right key according to the stimulus color. Hence, if a red stimulus is mapped to a left key, performance is faster and more accurate when it appears in the left location (i.e., corresponding location; left figure) than when it appears in the right location (i.e., noncorresponding location; right figure)

2009; Kubo-Kawai \& Kawai, 2010). Yet, exactly how to interpret the analyses and the extent to which the Simon effect functions can be attributed to temporal activation properties has not been resolved and remains somewhat ambiguous. In the present article, therefore, we examine in detail the process and justification for analyzing RT distributions and the assumptions that underlie interpretation of the obtained results. We focus on variants of the spatial Simon task and examine the primary empirical results, determining what conclusions can be reached on the basis of existing studies. The assessment of RT distribution analyses we provide should be of value not only to researchers studying the Simon effect, but also to those investigating a range of correspondence effects to which distribution analyses are applicable (e.g., picture-word categorization, Roelofs, 2008; Stroop interference tasks, Steinhauser \& Hübner, 2009; flanker tasks, Wylie et al., 2009).

\section{Group RT distributions}

In the typical Simon effect study, each participant performs a couple of hundred trials, which is an insufficient number for conducting meaningful analyses of the RT distributions for individual participants. Consequently, a procedure is used in which, for each participant, the distributions for corresponding and noncorresponding trial types are partitioned into quantiles (bins), typically somewhere between 4 and 10, and then group means are obtained for each quantile. The Simon effect for each quantile is then obtained as the difference in mean RT for the noncorresponding and corresponding trial types, and the Simon effect is plotted as a function of mean RT differences between these trial types for each bin. The pattern of change in effect size across the RT distribution is the result that is of most concern.

The procedure of dividing data into RT bins and then averaging across participants to obtain group quantiles is known as Vincentizing, or Vincent averaging, after work performed by Stella Vincent (1912). She averaged the learning curves of white rats in such a manner, arguing that this group curve "seems to be a truer representation of the learning process" (p. 16) than the individual curves because of its being unaffected by factors such as differences in number of trials for learning to occur. Vincent's procedure and rationale, in her words, were as follows:

In this experimental work an animal's trials were discontinued when the problem was learned or one period past such time. The numerical results of time and error for each animal were then divided into an equal number of serial groups, say ten, regardless of whether the learning had taken twenty or forty trials. In the one case there would be two numbers in each group and in the other four. The averages were then taken by groups and thus there was obtained for each animal a series of ten numbers. ... The quantities for all of the animals were then averaged in serial order and there was obtained in this way numerical data for plotting a curve in which the beginnings of the learning process corresponded as did also the second, third, and end stages. . . . A curve made in this way should represent the learning process in its successive stages with the individual variations eliminated in proportion to the number of animals used. (pp. 16-17)

In choice RT experiments, there are also large individual differences in the mean RT and variability of the RT distribution, leading to similar issues about representing the reaction process. Group distributions are of particular use when the individual participants' distributions are based on relatively few values, as in the typical choice RT task, and when a specific distribution is hypothesized and predictions can be made about effects of variables on different parameters (Thomas \& Ross, 1980). Ratcliff (1979) proposed that Vincent averaging could be applied to the RT distributions of individual participants to obtain a group distribution. He noted, in a manner analogous to Vincent's approach to learning curves, that RTs for individual participants can be ordered from shortest to longest and then divided into bins for which mean values are calculated. A group RT distribution can then be generated by averaging the values for each bin across participants. Ratcliff articulated the main point about this method, which is that "the group distribution should be thought of as representing the distribution of the average subject, just as averaged reaction time represents the reaction time of the average subject" (p. 449). 
Whereas Vincent (1912) gave only logical justification for her method of obtaining a group learning curve, Ratcliff (1979) went further in providing evidence for the validity and limitations of the Vincent averaging method for RT distributions. He showed that, for combinations of RT distributions differing only in the parameters that represent the time for some processing stage (e.g., exponential, logistic, normal, and Weibull distributions), the shape of the group distribution is the same as that of the individual participants' distributions, with the parameters being the mean of those for the individual distributions (see also Thomas \& Ross, 1980). Using Monte Carlo methods, Ratcliff also showed that when the method is applied to exGaussian distributions involving the convolution of normal and exponential distributions, which are often used in fitting empirical RT distributions, the group distribution also introduces little bias. Finally, he demonstrated empirically that fits of the convolution model to the group distributions from three experiments yielded parameter values similar to those of the mean parameter values from fits to individual-participant distributions. Thomas and Ross added to Ratcliff's work by showing that, for distributions for which the group function is not from the same family as the individual distributions, transformations of the RT data can render Vincentizing appropriate to perform - a method that they referred to as generalized Vincentizing.

\section{Distributional analyses of the Simon effect}

De Jong et al. (1994) introduced the method used to perform distributional analyses of the Simon effect. This method involves constructing separate Vincentized group RT distributions for corresponding and noncorresponding trials, as in Ratcliff's (1979) method, obtaining a Simon effect measure (i.e., $\mathrm{RT}_{\text {noncorresponding }}-\mathrm{RT}_{\text {corresponding) for }}$ each quantile, and then plotting the Simon effect as a function of mean RT for each quantile (sometimes called delta plots; e.g., Ridderinkhof, 2002a). De Jong et al. (1994) assumed that the activation, or priming, of the spatially corresponding response by the stimulus location occurred shortly after stimulus onset and then dissipated rapidly. The distribution analyses performed for their experiments were intended to support this assumption, and De Jong et al. (1994) stated, "The results of the distributional analyses of spatial SRC effects [Simon effects] in the experiments reported here almost certainly provide a reliable estimate of the actual time course of these effects" (p. 733, emphasis ours).

De Jong et al. (1994) studied situations that produce the Simon effect and ones that yield a reversal of it to favor noncorresponding locations, called the Hedge and Marsh reversal, but for now we restrict consideration to only those situations that produced the standard Simon effect. In Experiment 1, De Jong et al. (1994) included conditions in which participants responded to red and blue circles at left and right locations with left and right keypresses. In all conditions, each response key was assigned a color. In the variable-color and variable-name conditions, color and color name labels, respectively, appeared above the keys $1,500 \mathrm{~ms}$ before onset of the imperative stimulus; in the variable mapping condition, one key was designated as red and the other blue, and a cue indicated whether to respond on that trial with the key of the same or the opposite color. As is shown by the solid lines in the left panel of Fig. 2, with a compatible stimulus-response (S-R) color mapping, the Simon effect in all three conditions was largest at the shortest of five RT bins, with values ranging between 25 and $35 \mathrm{~ms}$, and then decreased to approximately zero by the last bin. De Jong et al.'s (1994) Experiment 2 showed a similar negative slope of the Simon effect function for two conditions in which the keys were labeled arbitrarily with $x x x x$ and ++++ (and the S-R color response mapping was arbitrary). Thus, labeling of response keys mattered little, and all five of the conditions with compatible or arbitrary mapping of colors to responses showed a Simon effect that decreased monotonically as RT increased.

Several factors prompted De Jong et al. (1994) to make the claim that the distribution analyses "almost certainly" provided a reliable estimate of the time course of the Simon effect. First, the functions matched closely predictions they derived from a two-process model with unconditional (automatic) and conditional (task-defined) components. In this model, the unconditional activation of the corresponding response occurs promptly and then dissipates, whereas the conditional activation of the response assigned by task instructions is independent of the time since stimulus onset (see Fig. 2, right panel). Evidence for the two components being distinct was provided through the Hedge and Marsh reversal, which we discuss in the next section. Second, De Jong et al. (1994) noted findings of Simon, Acosta, Mewaldt, and Speidel (1976) showing that the visual Simon effect was eliminated when a delay of 250 or $350 \mathrm{~ms}$ between stimulus onset and a go signal to respond was instituted.

The third, and main, reason for De Jong et al.'s (1994) conclusion that the distribution analyses provided reliable estimates of the time course was the outcome of Monte Carlo simulations that they reported in an Appendix. These simulations were conducted to establish conditions that might yield decreasing Simon effect functions for reasons other than temporal activation properties. Specifically, the simulations evaluated whether the pattern could be generated solely from large trial-to-trial variability of the Simon effect. De Jong et al.'s (1994) simulations used two exGaussian distributions (convolutions of normal and expo- 


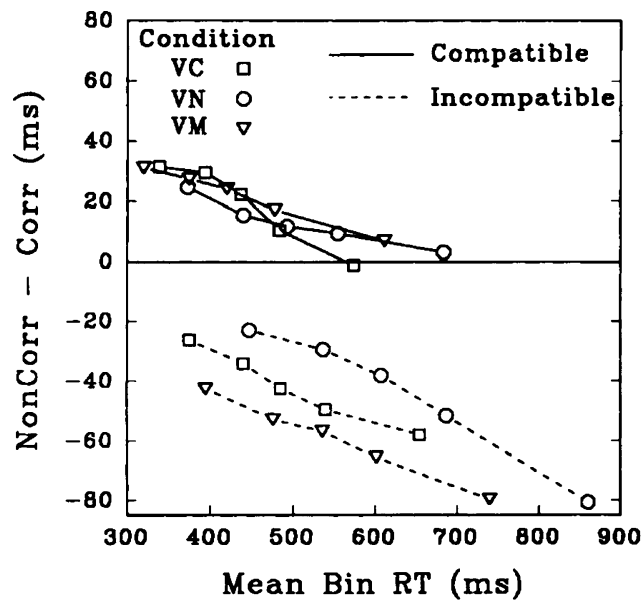

Fig. 2 Left panel: Spatial noncorresponding (Noncorr) minus corresponding (Corr) reaction times (RTs, in milliseconds) for the three conditions $(\mathrm{VC}=$ variable color, keys labeled with colors; $\mathrm{VN}=$ variable name, keys labeled with color names; $\mathrm{VM}=$ variable mapping, keys labeled with the same neutral color) and two mappings (compatible or incompatible mapping of stimulus color to response color) in De Jong et al. (1994) Experiment 1, as a function of RT bin. Right panel: Predictions of De Jong et al.'s (1994) dual-process

nential distributions). Nine simulations were run, for three levels of variance $\times$ three different combination rules representing situations in which the Simon effect is due entirely to (1) a benefit on corresponding trials, (2) a cost on noncorresponding trials, or (3) both. De Jong et al. (1994) summarized the simulation results as follows:

The results . . demonstrate clearly that the experimentally obtained result of a strong decrease in the Simon effect from fast to slow bins can reasonably be attributed to random variability of the Simon effect only if (a) the Simon effect is due almost exclusively to an advantage for corresponding $\mathrm{S}-\mathrm{R}$ positions, and (b) the standard deviation of the overall Simon effect greatly exceeds its mean.” (p. 749)

Because "both conditions seem quite unrealistic" (p. 749), they concluded that "the results of the distributional analyses would seem to be explained most easily and most naturally by the notion that spatial SRC effects [Simon effects] are transient" (p. 750).

Zhang and Kornblum (1997) challenged this conclusion of De Jong et al. (1994), providing evidence that, in their words, "the distributional analysis is a simple computational procedure that reflects fundamental statistical properties of the underlying reaction time distributions and their interrelationships and that De Jong et al.'s time-course assumptions precluded at least half of these interrelationships" (p. 1543). Zhang and Kornblum reported a mathematical proof in which they showed that a negative slope of the type obtained by De Jong et al. (1994) occurs

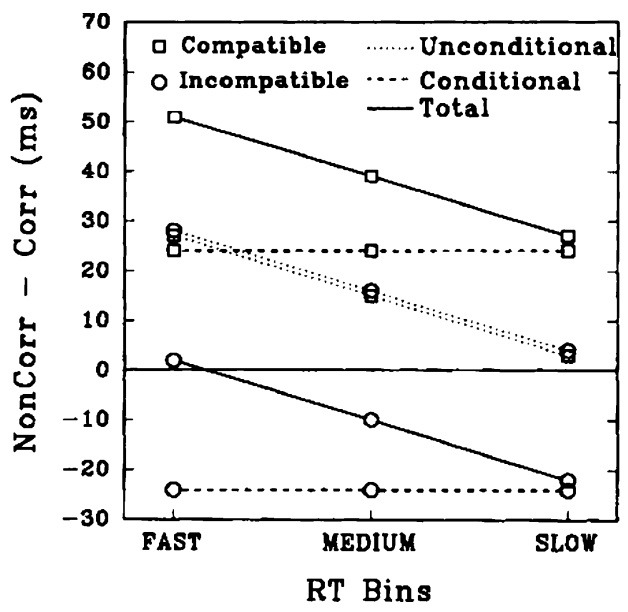

model, with the separate contributions of the conditional and unconditional components illustrated for compatible and incompatible mappings of stimulus colors to keys. From De Jong et al. (1994), Conditional and unconditional automaticity: A dual-process model of effects of spatial stimulus-response correspondence. Journal of Experimental Psychology: Human Perception and Performance, 20, 731-750. Reprinted with permission of the American Psychological Association

when the variance of the RT distribution for corresponding trials is larger than that for noncorresponding trials. If the variance relation is the opposite, the Simon effect function will show a positive slope, increasing across the RT distributions.

To illustrate that an increasing function is a plausible outcome, Zhang and Kornblum (1997) reported data from Kornblum (1994) using a nonstandard procedure that showed a positive slope for the Simon effect. In Kornblum's experiment, a left or right keypress was made to the color (green or blue) of a stimulus presented in the left, right, upper, or lower part of a rectangle. The location of the color patch was irrelevant, as was the identity of a letter string shown at the center of the rectangle. For a pure trial block, the colors appeared in the upper or lower half of the rectangle on one third of the trials (neutral trials) and in the left or right half of the rectangle on the remaining two thirds, with the location corresponding to that of the response on half of those trials and not on the other half. Similar results were obtained for a mixed block, in which those trials with S-R location correspondence/noncorrespondence were mixed with other trials containing only stimulus-stimulus correspondence/noncorrespondence (colors in upper or lower locations, with irrelevant color words in the center). In both pure and mixed blocks, the corresponding trials showed less variability than the noncorresponding trials, and the Simon effect increased from 10 to $20 \mathrm{~ms}$ at the shortest of five RT bins up to 55-65 ms at the longest. Additionally, Zhang and Kornblum analyzed the conditions simulated by De Jong et al. (1994) and 
demonstrated that the simulation results were as predicted by their variance proof.

To summarize, the studies of De Jong et al. (1994) and Zhang and Kornblum (1997) indicate the following. Decreasing distribution functions (negative slopes) for the Simon effect are consistent with the hypothesis of rapid activation of the corresponding response followed by dissipation of this activation. However, the more basic relation in the data producing this pattern is larger variability for the corresponding RT distribution than for the noncorresponding RT distribution, and this difference in variability could occur not only as a consequence of the time course of activation, but also for other reasons. In some variants of the Simon task, it is possible to reverse the relative variability of the two distributions and obtain functions for which the Simon effect increases as RT increases. With these points in mind, we examine the empirical results of distribution analyses for the Simon effect, emphasizing the variety of distribution functions that have been obtained. We interpret the results primarily in terms of the time course of activation. Following this examination, we return to the question of whether the distribution functions do, in fact, primarily reflect temporal activation properties by examining results obtained with other measures for assessing the time course of processing.

\section{Time course of the standard Simon effect: evidence for activation-dissipation}

We begin by considering the distribution functions for the standard left-right Simon effect obtained with visual stimuli and keypress responses, as examined by De Jong et al. (1994). After doing so, we examine the generalizability of the results to other variations of the Simon task and evaluate alternative theoretical accounts of the distribution functions that have been proposed. Many of the key results are summarized in Table 1.

Decreasing functions for bimanual keypresses to visual left-right stimuli

A widely used form of the visual Simon task is to present stimuli of one of two colors in a left or right location, to which a left keypress with the left index finger or a right keypress with the right index finger is to be made on the basis of the stimulus color. As was noted, De Jong et al. (1994) found that this type of situation yielded a Simon effect that decreased monotonically across the RT distribution (negative slope) when the mapping of stimulus colors to response keys was (1) arbitrary (the keys were labeled with $\operatorname{xxxx}$ or ++++ ) or (2) compatible with physical or verbal color labels used to designate the keys (see Fig. 2, left panel). The conditions examined by De Jong et al. (1994) were a little different from those of the basic leftright Simon task, in which response keys are typically unlabeled and designated only as left and right. Thus, a first question is whether the negatively sloped Simon effect function is obtained reliably for the visual Simon task with left and right keypresses (see Fig. 1).

For studies in which keypresses were made with the left and right index fingers to lateralized stimuli of one of two colors, the Simon effect has typically been largest at the shortest one or two RT bins and then decreased across the remaining bins, as in De Jong et al.'s (1994) study (e.g., Kubo-Kawai \& Kawai, 2010; Proctor, Yamaguchi, Zhang, \& Vu, 2009, location control condition of Experiment 1; Vu \& Proctor, 2008, pure Simon effect condition of Experiment 2). For example, in Proctor et al.'s (2009) study, the Simon effect for the location control condition was approximately $35 \mathrm{~ms}$ at the first of $4 \mathrm{RT}$ bins and decreased to slightly less than $0 \mathrm{~ms}$ at the longest RT bin. A study by Davranche and McMorris (2009), in which the keys were pressed with the left and right thumbs, found a small increase from 29 to $38 \mathrm{~ms}$ across the first three of $10 \mathrm{RT}$ bins, but then the Simon effect decreased to $2 \mathrm{~ms}$ by the last RT bin.

This pattern of increase followed by decrease has been reported consistently for studies in which a neutral noise stimulus was presented in the position opposite the imperative stimulus. Wascher, Schatz, Kuder, and Verleger (2001, Experiment 1) obtained such a result when the relevant stimulus dimension was letter identity, $A$ or $B$, and a noise stimulus of three horizontal lines similar in size and luminance to the letters appeared in the location opposite that occupied by the target stimulus. With the hands in a normal position, the Simon effect increased from $23 \mathrm{~ms}$ at the first of nine bins to $35 \mathrm{~ms}$ at the third bin and then decreased to $15 \mathrm{~ms}$ at the final bin. Using the same task, Buhlmann, Umiltà, and Wascher (2007) obtained similar results for their condition in which left and right index fingers pressed left and right response buttons, as did Vallesi, Mapelli, Schiff, Amodio, and Umiltà (2005) for a task that required a left-right keypress to a red/black or green/black checkerboard (with a white/black checkerboard appearing in the opposite location). The temporal activation account provides a straightforward interpretation of these results: The presence of a noise stimulus in the location opposite the stimulus may delay activation of the response code corresponding to the stimulus location, shifting the peak activation relative to processing of the relevant stimulus dimension to a later time.

A result to note is the Simon effect distribution function that occurs when, in the Hedge and Marsh (1975) variant of the Simon task, the mapping of stimulus color to response color is incompatible. For that condition, keys are labeled 
Table 1 Overview of the most important parameters relating to the Simon effect (SE) for physical locations and its time course across studies discussed in this article

\begin{tabular}{|c|c|c|c|c|c|c|c|}
\hline Stimulus & Response & Study & SE (ms) & SE Time Course & $\begin{array}{l}\text { SE Range } \\
\text { (ms) }\end{array}$ & No. of Bins & $\begin{array}{l}\text { RT Range } \\
\text { (ms) }\end{array}$ \\
\hline \multicolumn{8}{|l|}{ Standard Simon Effect } \\
\hline \multirow[t]{8}{*}{ Horizontal colors } & \multirow{8}{*}{$\begin{array}{l}\text { Horizontal left/right } \\
\text { hands }\end{array}$} & De Jong et al. (1994) & & & & & \\
\hline & & Exp. 1 (compatible) & 18 & decreasing & $33-0$ & 5 & $350-580^{*}$ \\
\hline & & $\begin{array}{l}\text { Kubo-Kawai and Kaw } \\
\text { (2010) }\end{array}$ & & & & & \\
\hline & & Exp. 1 (young group) & 11 & $\begin{array}{l}\text { stable then } \\
\text { decreasing }\end{array}$ & $20--9$ & 4 & $320-490 *$ \\
\hline & & Proctor et al. (2005) & & & & & \\
\hline & & Exp. 1 (young group) & 7 & decreasing & $28--26$ & 4 & $360-650^{*}$ \\
\hline & & Vallesi et al. (2005) & & & & & \\
\hline & & Exp. 1 & 21 & decreasing & $33--8$ & 5 & $344-589$ \\
\hline \multirow[t]{2}{*}{ Bilateral horizontal letters } & \multirow{2}{*}{$\begin{array}{l}\text { Horizontal left/right } \\
\text { hands }\end{array}$} & Wascher et al. (2001) & & & & & \\
\hline & & Exp. 1 & 25 & $\begin{array}{l}\text { increasing then } \\
\text { decreasing }\end{array}$ & $33-15$ & $\begin{array}{l}10 \text { (last bin } \\
\text { discarded) }\end{array}$ & $370-570^{*}$ \\
\hline \multicolumn{8}{|c|}{ Other Simon Effects with Physical Locations } \\
\hline \multirow[t]{2}{*}{ Bilateral horizontal letters } & \multirow{2}{*}{$\begin{array}{l}\text { Crossed horizontal } \\
\text { left/right hands }\end{array}$} & Wascher et al. (2001) & & & & & \\
\hline & & Exp. 1 & 32 & increasing & $14-52$ & $\begin{array}{l}10 \text { (last bin } \\
\text { discarded) }\end{array}$ & $360-620^{*}$ \\
\hline \multirow[t]{4}{*}{ Auditory tones on horizontal side } & \multirow{4}{*}{$\begin{array}{l}\text { Horizontal left/right } \\
\text { hands }\end{array}$} & Proctor and Shao (201 & & & & & \\
\hline & & Exp. 1 & 35 & $\begin{array}{l}\text { increasing } \\
\text { then stable }\end{array}$ & $14-40$ & 5 & $310-610^{*}$ \\
\hline & & Wascher et al. (2001) & & & & & \\
\hline & & Exp. 2 & 46 & increasing & $25-60$ & $\begin{array}{l}10 \text { (last bin } \\
\text { discarded) }\end{array}$ & $290-540 *$ \\
\hline \multirow[t]{2}{*}{ Vertical colors } & \multirow{2}{*}{$\begin{array}{l}\text { Vertical left/right } \\
\text { hands }\end{array}$} & Vallesi et al. (2005) & & & & & \\
\hline & & Exp. 1 & 29 & stable & $23-35$ & 5 & $328-547$ \\
\hline \multirow[t]{4}{*}{ Vertical colors (mixed mapping) } & \multirow{4}{*}{$\begin{array}{l}\text { Vertical left/right } \\
\text { hands }\end{array}$} & De Jong et al. (1994) & & & & & \\
\hline & & $\begin{array}{l}\text { Exp. } 4 \text { (mixed } \\
\text { mapping) }\end{array}$ & $40^{*}$ & decreasing & $60-35$ & 5 & $350-610^{*}$ \\
\hline & & $\begin{array}{l}\text { Valle-Inclán and Redor } \\
\text { (1998) }\end{array}$ & & & & & \\
\hline & & Exp. 1 & 77 & decreasing & $101-37$ & 5 & $\mathrm{~N} / \mathrm{A}$ \\
\hline \multirow[t]{2}{*}{ Vertical horizontal colors } & \multirow{2}{*}{$\begin{array}{l}\text { Horizontal } \\
\text { left/right hands }\end{array}$} & Proctor et al. (2003) & & & & & \\
\hline & & Exp. 1 & 16 & increasing & $6-41$ & 10 & $300-650 *$ \\
\hline
\end{tabular}

Note. Values designated with asterisks are approximated values

with the same colors as the two alternative stimuli, and participants are told to press the key with the color opposite to that of the stimulus. The result is a negative Simon effect, favoring the spatially noncorresponding response, the finding known as the Hedge and Marsh reversal. De Jong et al. (1994) included incompatible color-mapping conditions in their study and found that the Hedge and Marsh reversal of the Simon effect increased as RT lengthened. Hedge and Marsh attributed the reversal to application of a respond-opposite rule to the irrelevant location feature, as well as to the relevant color feature, and De Jong et al. (1994) included this account in their model. They proposed that this conditional component is indepen- dent of the amount of time after stimulus onset at which it is performed (see Fig. 2, right panel), whereas the unconditional activation of the corresponding response decreases over time. Thus, their model predicts the increasing time course of the negative Simon effect, because the tendency to make the corresponding response decreases over time, leaving the conditional activation of the opposite response location as the only factor when RT is long.

To summarize, in several variations of basic left-right Simon tasks, with bimanual keypress responses, a decrease in the Simon effect from the short RT bins to the long RT bins is reliably obtained. This is sometimes accompanied 
by an initial increase across the first few RT bins, which was not evident in De Jong et al.'s (1994) original study. Also, the increasing function for the reversed Simon effect obtained with an incompatible mapping of stimulus color to response color is consistent with decreasing activation of the corresponding response across time. On the whole, then, the results are in agreement with the hypothesis that the distribution functions obtained for the Simon effect and its reversal in the Hedge and Marsh paradigm are due to rapid activation of the corresponding response, followed by a decrease in activation.

Dissipation of the activation: decay or suppression?

Although De Jong et al. (1994) described activation of the corresponding response as "unconditionally automatic" (p. 745) and transient, they stated, "whether this phenomenon [rapid dissipation] reflects a passive decay of automatic priming ... or an active process of suppression, remains to be determined" (p. 745). Hommel (1993) was similarly noncommittal with regard to his temporal overlap account of the Simon effect, according to which the size of the Simon effect decreases as the temporal distance between the coding of the irrelevant stimulus location and that of the relevant stimulus increases, stating that the weakening of the irrelevant code over time could be due to decay or suppression. However, Hommel (1994b) provided evidence that passive decay, rather than suppression, is the cause of the decrease. He varied the percentage of noncorresponding trials included in trial blocks $(50 \%$ vs. $75 \%$ in Experiment 1; $20 \%$ vs. $80 \%$ in Experiment 2), with the idea that strategic suppression would be more likely to occur when noncorresponding trials predominate than when they do not. The time to identify the relevant stimulus feature was also varied across trial blocks by making the relevant dimension of high or low discriminability in Experiment 1 and having relevant letter identity features appear all at once or gradually in Experiment 2. Hommel (1994b) predicted that, if active suppression were occurring, the Simon effect would interact with the frequency and discriminability manipulations, because a delay in identification of the relevant feature would allow more time for suppression to be applied. In contrast, the decay account predicts no such interaction, because time is the only critical factor. Although the Simon effect decreased when identification of the relevant feature was made more difficult and when noncorresponding trials predominated, the results showed no sign of an interaction, thus supporting the decay account.

Ridderinkhoff subsequently conducted several studies that were interpreted as supporting a suppression account. According to his activation-suppression hypothesis (see Ridderinkhof 2002a), the spatial stimulus dimension auto- matically triggers a corresponding response through an unconditional pathway. An active suppression would be eventually engaged to contain such automatic activation. Importantly, the stronger this suppression, the faster and more efficient it will be, leading to a reduced level of interference already from the first bins. Ridderinkhof (2002b) described this strategic suppression more generally as "cautiousness."

Ridderinkhof (2002b) had participants perform a Simon task similar to that of Hommel (1994b), with blocks in which either $25 \%$ or $75 \%$ of the trials were noncorresponding, but he focused on analyses of RT distributions. According to the activation-suppression hypothesis, when noncorresponding trials predominate, thus biasing the response on any given trial to be noncorresponding, participants adopt a more cautious response strategy and suppress the tendency to allow automatic activation of the corresponding response to direct behavior. Ridderinkhof's (2002b) results showed that the Simon effect was smaller when the percentage of noncorresponding trials was high ( $-9 \mathrm{~ms})$ than when it was low $(34 \mathrm{~ms})$, but the Simon effect decreased at the same rate for both conditions as RT lengthened. This lack of influence of the percentage manipulation on the decreasing Simon effect distribution function is consistent with Hommel's (1994b) results described in the first paragraph of this section and with a passive decay interpretation.

However, Ridderinkhof (2002b) decomposed the distribution functions into trials that followed correct or incorrect responses and found an interaction of the percentage manipulation and sequence: When $25 \%$ of the trials were noncorresponding, the slope of the Simon effect function was more negative for posterror trials than for postcorrect trials, but when $75 \%$ of the trials were noncorresponding, the slopes were similar for posterror and postcorrect trials. In the latter case, there also was little impact of the percentage or sequence variable on the Simon effect functions for error rates across the distribution, but when $25 \%$ of the trials were noncorresponding, the preponderance of error responses at the shortest RT bin were to respond "corresponding" when the correct response was noncorresponding, especially if the response for the prior trial was correct. Ridderinkhof (2002b) interpreted these results as support for the activation-suppression account.

In another study, Ridderinkhof (2002a) used additional methods to evaluate this account. Participants performed under mixed-task conditions in which $75 \%$ of the trials were of a Simon task in which a left or right keypress response was made to the black or white color of a diamond shape presented in a left or right location. The remaining $25 \%$ of the trials were either other Simon trials for which a left or right keypress was to be made to the circle or square shape of a gray stimulus presented in a left or right location 
or compatibly mapped spatial trials for which the spatially corresponding response was to be made to a gray stimulus in the left or right position. Thus, in the former case, stimulus location was always task irrelevant, whereas in the latter case, stimulus location was occasionally relevant. Ridderinkhof (2002a) reasoned that the Simon effect will decline across the RT distribution when stimulus location is always irrelevant, since it is beneficial to constantly apply a high degree of suppression throughout task performance. When the Simon task is intermixed with location-relevant trials, less suppression will occur, and the Simon effect will not decline over the RT distribution, since suppression of stimulus location would harm performance on locationrelevant trials. As was expected, the results showed a declining function, along five bins, when location was never relevant and an increasing function when location was occasionally relevant, which Ridderinkhof (2002a) interpreted as suggesting that the amount of suppression applied to direct response activation depended on task context.

In the same study, participants first performed blocks in which all the trials were the black-white Simon task. For analysis of these data, Ridderinkhof (2002a) used a median split to create two groups, those with smaller and larger Simon effects. The former group showed a declining effect function, whereas the latter group displayed a relatively stable effect across the RT distribution. Variability in the Simon effect appeared to be due mainly to differences in the pattern of Simon effects at the longest RTs, rather than to an overall increase in Simon effects across the entire distribution, consistent with the individual differences being associated with suppression. In another analysis of the pure Simon task data, Ridderinkhof (2002a) examined sequential effects and found, as have others (e.g., Stürmer, Leuthold, Soetens, Schröter, \& Sommer, 2002), that the Simon effect was reduced following a noncorresponding trial. According to him, this reduction occurred because the conflicting activation led to a "tuning up" of the suppression mechanism that was still effective during the following trial.

Ridderinkhof and colleagues found additional affirmation for the activation-suppression hypothesis from neurological studies. For example, Forstmann, van den Wildenberg, and Ridderinkhof (2008) found that activation in the right inferior frontal cortex, an area associated with inhibition (see Aron, Robbins, \& Poldrack, 2004), was also associated with the declining component of the Simon effect for longer RTs (see also Band, Ridderinkhof, \& van der Molen, 2003; Burle, Possamaï, Vidal, Bonnet, \& Hasbroucq, 2002).

The Simon effect RT distributions of older adults and persons with clinical disorders, as compared with those of healthy younger adults, provide further evidence consistent with the activation-suppression hypothesis. For instance, Juncos-Rabadán, Pereiro, and Facal (2008) asked participants in varying age groups to respond to the direction of a lateralized pointing arrow, ignoring its location on the screen (left or right). Their results showed that the function of the Simon effect across RTs gradually changed from a negative to a positive slope with increasing age (Fig. 3, left panel). Young participants (19-26 years) showed a decreasing time course (the magnitude of the effect was $42 \mathrm{~ms}$ at the first bin and then drifted to $22 \mathrm{~ms}$ in the last of four bins), older adults (50-59 and 60-69 years) displayed a stable effect across RT bins, and the oldest adults (70-82 years) showed an increase in effect size from $92 \mathrm{~ms}$ in the first bin to $122 \mathrm{~ms}$ in the last bin. The authors claimed that the agerelated differences in Simon effect magnitude and its time course are a consequence of a reduced ability of older adults to suppress response activation.

Castel, Balota, Hutchison, Logan, and Yap (2007, Experiment 1) had younger and older adults perform the same task as that in Juncos-Rabadán et al.'s (2008) study, with inclusion of a neutral condition in which the arrow stimuli were presented centrally. As was hypothesized, and in line with Juncos-Rabadán et al., the Simon effect increased across bins for the older adults and decreased for the younger ones. The latter result was replicated in a follow-up experiment (Experiment 1B) in which stimuli were perceptually degraded by reducing their luminance level (Fig. 3, right panel). Such consistency of findings led the authors to conclude that the decreasing versus increasing pattern is uniquely related to attention and response control processes, and not to the visual discriminability for peripheral stimuli. In Experiment 2, Castel et al. also tested Alzheimer's disease (AD) patients, and the results were similar to those for healthy older adults: The Simon effect of mild $\mathrm{AD}$ patients increased over time. In contrast to these studies showing a positive slope for older adults' Simon effect functions, for the standard color Simon task, Proctor, Pick, Vu, and Anderson (2005) and Vu and Proctor (2008) found a decrease of the Simon effect across RT bins for healthy older adults that was not significantly different from that for younger adults. These results suggest that the difficulty in ignoring/suppressing activation from irrelevant stimulus location evidenced by older adults in other studies may be a consequence of the relevant stimulus dimension also conveying spatial information.

More recently, Wylie, Ridderinkhof, Bashore, and van den Wildenberg (2010) had participants perform a version of the Simon task in which left or right buttonpresses with the thumbs were made on hand-held grips on the basis of the color of a left or right stimulus. Patients with Parkinson's disease (PD), which is associated with a deficit in inhibition of motor activation, were divided into three groups ranked by level of disease severity. The group with the most advanced PD symptoms showed no decline in the Simon effect across the distribution, the group with moderate symptoms showed a slight decline from 37 to $17 \mathrm{~ms}$, and 
Juncos-Rabadán et al. (2008)

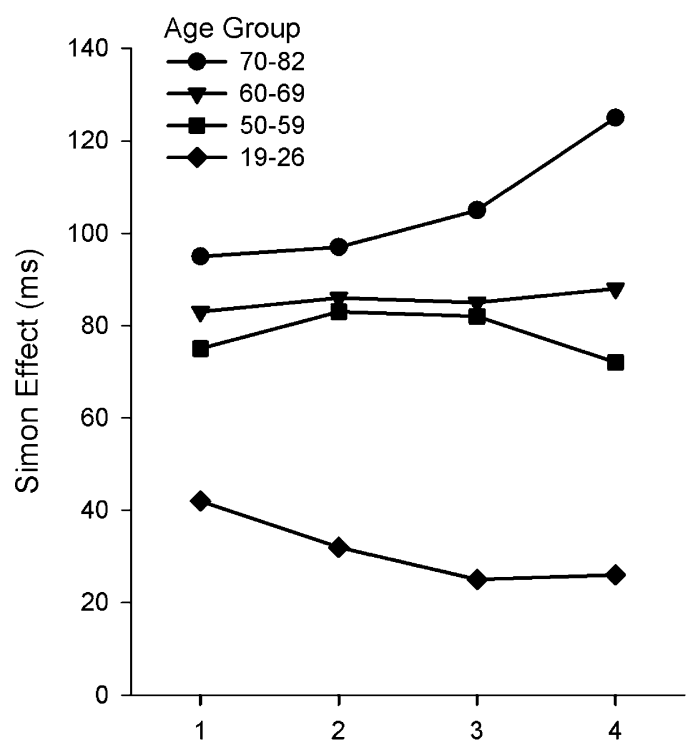

Castel et al. (2007)

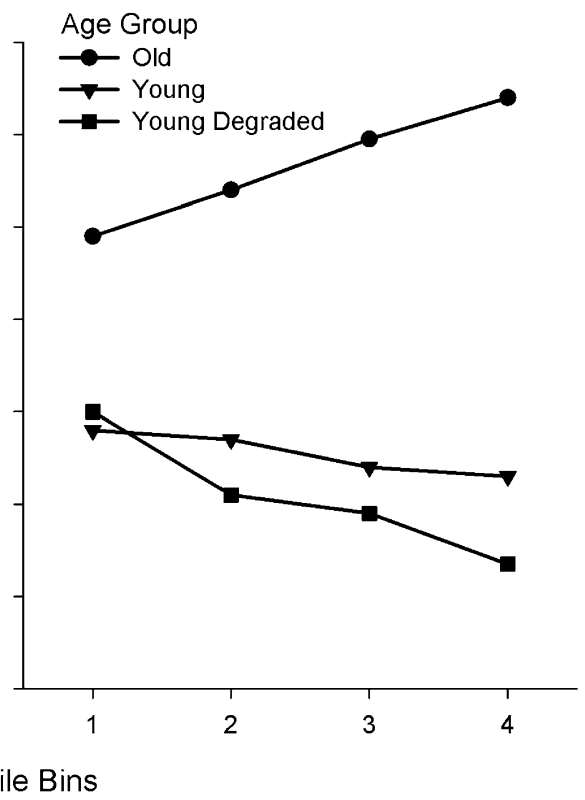

Fig. 3 The Simon effect magnitude (in milliseconds) for each bin as shown in the studies of Juncos-Rabadán et al. (2008; left panel, legend refers to age ranges in years) and Castel et al. (2007; right panel, legend refers to healthy old and young adults, the latter performing the task with or without the stimuli being degraded). Left panel from Juncos-Rabadán et al. (2008), Cognitive interference and aging: Insights from a spatial stimulus-response consistency task. Acta
Psychologica, 127, 237-246. Reprinted with permission of Elsevier B. V. Right panel from Castel et al. (2007), Spatial attention and response control in healthy younger and older adults and individuals with Alzheimer's disease: Evidence for disproportionate selection impairments in the Simon task. Neuropsychology, 21, 170-182. Reprinted with permission of the American Psychological Association those with only minor symptoms showed a declining function comparable to that of healthy controls (from around $35 \mathrm{~ms}$ in the first bin to $-10 \mathrm{~ms}$ in the final bin).

To summarize, although the issue is not fully settled and some evidence favors a decay account of the declining Simon effect functions across the RT distribution, on balance, the results from neurological and individualdifference studies are generally consistent with an activation-suppression account. However, as we discuss next, there are many cases in which Simon effect functions for young adults increase or remain stable across the RT distributions, and both a general decay explanation and the activation-suppression hypothesis have little to say about what types of stimuli and responses sets per se will lead to decreasing versus increasing functions.

\section{The time course of other Simon effects with physical locations}

Hommel's (1993, 1994a) temporal overlap account and De Jong et al.'s (1994) two-process model imply that rapid activation of the corresponding response and dissipation of activation should occur in a variety of situations, since the activation is "automatic" in the sense that it is irrelevant to the task and, thus, should be suppressed or allowed to quickly decay. De Jong et al.'s (1994) model captures this property by calling the route that produces the decreasing activation "unconditional," and the property is also in general agreement with Kornblum et al.'s (1990) model of S-R compatibility effects, according to which automatic activation of the corresponding response occurs when stimulus and response dimensions overlap, or are similar, regardless of task instructions. An implication is that the decreasing effect pattern should be evident for other variants of the Simon effect. Yet several findings indicate that such a decreasing effect function is not a common feature of all Simon tasks with physical locations (see the bottom half of Table 1).

Crossed hands and acoustic stimuli

Wascher et al. (2001) proposed that, instead of automatic activation of the corresponding response being a general property of tasks with dimensional overlap, it is limited to tasks in which the dorsal visuomotor pathway provides a basis for responding: "Assuming that a highly efficient visuospatial network is necessary to enable abilities like 
reaching or grasping, activation should be restricted to the visual system" (p. 734). According to Wascher et al., the functions of this "highly efficient visuomotor system might form the basis for the natural intrahemispherical coactivation of perceptual and motor areas in the standard conditions of the visual Simon task" (p. 739). More specifically, automatic activation should be the cause of the Simon effect only for situations in which the response can be executed with the effector (hand) corresponding to the cerebral hemisphere in which the stimulus would initially be processed. Wascher et al. attributed Simon effects that occur for other situations to another mechanism, "interference between codes during translation of stimulus into response coordinates" (p. 731), which would increase the variability for the RT distribution on noncorresponding trials, as demonstrated originally by Zhang and Kornblum (1997). Wascher et al. reported results generally consistent with this distinction between mechanisms, showing that the decreasing functions were not obtained when responding with crossed hands to visual stimuli or when responding with crossed or uncrossed hands to auditory stimuli.

In Wascher et al.'s (2001) Experiment 1, as was described earlier, participants made left or right buttonpresses depending on whether a target in a left or a right location was the letter $A$ or $B$; a noise stimulus (horizontal lines) occupied the alternative location. Participants performed in some trial blocks with a normal hand placement, for which the left response was made with the left hand and the right response with the right hand, and in others with a crossed-hands placement, for which the right hand operated the left key and the left hand the right key. Consistent with prior research (e.g., Wallace, 1971), the overall Simon effect with respect to the spatial correspondence between target and response locations was of similar size for the two hand placements. However, only the normal hand placement showed the decreasing function of the Simon effect across the RT distribution; with the crossed-hands placement, the Simon effect increased across the entire distribution (see Fig. 4, left side). Lateralized readiness potentials (LRPs), event-related brain potentials that indicate preparation to respond with the left or right hand, were recorded during the experiment. In such studies, it is generally assumed that neural activation in the motor cortex is strongly related to response activation on the contralateral side. They showed an asymmetry in motor cortex activation such that there was temporarily greater cortical activation on the side contralateral to the target stimulus (the so-called Gratton dip; Gratton, Coles, Sirevaag, Eriksen, \& Donchin, 1988). Of importance, this asymmetry was greater in the uncrossed-hands condition than in the crossed-hands

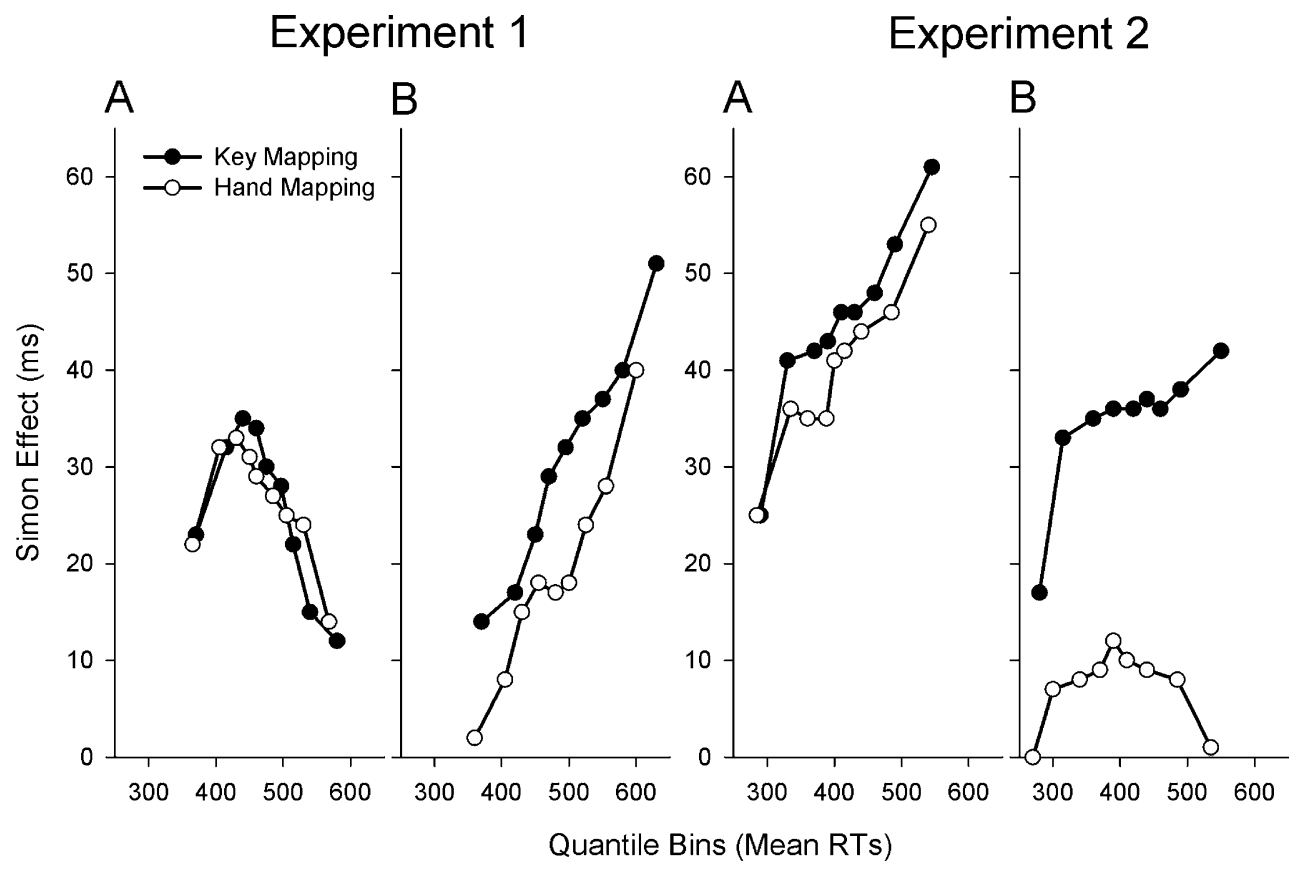

Fig. 4 Data from Wascher et al.'s (2001) Experiments 1 and 2. The Simon effect magnitude plotted against the mean base reaction time for corresponding and non corresponding trials in each quantile. Key mapping and hand mapping refer to whether instructions were in terms the mapping of the relevant stimulus dimension to left/right response keys or left/right hands. In Experiment 1, the stimuli were visual, and the relevant dimension was letter identity; in Experiment 2, the stimuli were auditory, and the relevant dimension was tone pitch. From Wascher et al. (2001), Validity and boundary conditions of automatic response activation in the Simon task. Journal of Experimental Psychology: Human Perception and Performance, 27, 731751, 2001. Reprinted with permission of the American Psychological Association 
condition, suggesting that activation was stronger when the hands were in their normal positions.

In Experiment 2, Wascher et al. (2001) reported evidence that they interpreted as indicating that automatic activation is restricted to visual stimuli. The stimuli were 1000- and $1500-\mathrm{Hz}$ tones, presented from left and right loudspeakers, that were to be classified with left and right keypresses. With the uncrossed-hands placement, the Simon effect was $25 \mathrm{~ms}$ in the first RT bin, a value like those in the visual studies. But instead of decreasing, the effect increased across the RT distribution, reaching a value of $55-60 \mathrm{~ms}$ in the final of nine RT bins. The increasing pattern was not as clear when participants performed with the crossed hands placement, although there was little sign of a decrease in effect size across the RT distribution, either (see Fig. 4, right side). Proctor and Shao (2010) replicated the pattern of increasing auditory Simon effect at longer RT bins, and their results showed little difference in the distribution functions for uncrossed and crossed hands, confirming that hand placement is of little consequence for the auditory Simon task.

Wascher et al. (2001) provided the following interpretation of the totality of their results in the summary for their article: "There are some prerequisites for the initiation of sensorimotor activation processes: (a) activation is a characteristic of the visual sense because it is part of the grasping system, and (b) the required movement has to be anatomical" (p. 749). The hypothesis that visuomotor activation depends on correspondence between stimulus position and spatial anatomical status of the effector implies that a decreasing function for the visual Simon effect should occur only when there is correspondence with the left- and right-hand distinction and responses are coded in terms of this distinction. Already in Wascher et al.'s study, results counter to this implication were evident in their Experiment 3. In this experiment, which used a visual Simon task similar to that in Experiment 1, with the main difference being inclusion of a condition in which there was no noise stimulus opposite the target in addition to the condition in which there was a noise stimulus, "different from Experiment 1, the S-R correspondence effect generally decreased for the very slowest responses" (p. 745).

\section{Action goals}

Action goals can be dissociated from anatomical effectors in the Simon task not only by crossing the hands, but also by having each hand hold a stick that is used to press the contralateral response key (Riggio, Gawryszewski, \& Umiltà, 1986). Buhlmann et al. (2007) provided evidence that the visual Simon effect shows an increasing function when responses are coded in terms of the location in space where an action achieves its effect but a decreasing function when responses are coded according to the anatomical mapping of the acting hand. In three experiments, participants responded to lateralized letters $(A$ or $B)$, as in Wascher et al.'s (2001) study. In the pure blocks conditions, participants pressed left/right keys (Experiments 1 and 2) or a central key (Experiment 3) with the left and right index fingers, respectively. In a separate trial block, left/right keys were operated with wooden sticks that were crossed such that the stick held in the left hand pressed the right key and vice versa. Consistent with Wascher et al.'s study, the finger responses showed a decreasing time course (along nine RT bins) of the Simon effect and an initial deflection in LRPs, whereas the crossed stick responses showed an increasing Simon effect, relative to the key locations, and a smaller LRP motor component.

Of more interest are the results from mixed conditions, in which one response was made with a fingerpress and the other with a stick held by the hand contralateral to the response key. This was accomplished by using an apparatus in which both hands were placed on platforms, with the finger of one hand used to press a key on the respective platform and the other hand used to hold a stick that pressed a key on the tabletop, located below the platform. When the hand placement was such that the stick operated the key on the same side as the finger response (Experiment 1 ), so that the response goals were not distinguished by left-right location but the effectors were, both the finger and stick responses showed decreasing Simon effect functions, relative to the hand. This result implies that both responses were coded in terms of the anatomical distinction. In contrast, when the hands were positioned such that the finger response was made at the location under that of the hand operating a contralateral key (e.g., the left hand on the platform operated the right key, and the right hand the left key), the Simon effect was relative to the left-right location of the response goal and showed an increasing time course for fingers and sticks. This finding implies coding of responses in terms of the goal location, which, according to Wascher et al. (2001), would situate the Simon effect in the translation process.

These results with the crossed stick responses are generally in agreement with Wascher et al.'s (2001) account of two dissociable mechanisms underlying the Simon effect. Visuomotor activation, as indicated by a decaying time course and an early deflection in LRP, was found if a spatial anatomical mapping was shared between stimuli and responses. In contrast, when responses were selected according to their goal locations, the cognitive mechanism drove behavior, showing an increasing effect function for RTs indicative of greater variability on noncorresponding than on corresponding trials (Zhang \& Kornblum, 1997). 
Vertically oriented stimulus and response sets

Increasing functions According to Wascher et al.'s (2001) account, when stimuli and responses vary along the vertical dimension (up and down locations), the Simon effect is not due to automatic activation (because there is no direct correspondence of up and down stimulus locations with left and right hands), and therefore, the vertical Simon task should show a flat or an increasing effect function. Stürmer et al. (2002, Experiment 3) obtained results consistent with this prediction in an experiment that used vertical $S-R$ arrangements as a control procedure for measuring LRPs. Participants responded to a square or a diamond stimulus, displayed in an upper or lower location on the screen, by pressing an upper or lower key on a keypad, which was in a normal position on the table. The Simon effect was large for trials following corresponding trials and absent for those following noncorresponding trials, a result that has been replicated several times (e.g., Hommel, Proctor, \& Vu, 2004). Of importance, the effect following corresponding trials increased from about $40 \mathrm{~ms}$ at the shortest of $20 \mathrm{RT}$ bins to more than $60 \mathrm{~ms}$ at the last few bins, whereas the effect following noncorresponding trials drifted from 0 to slightly negative $(<10 \mathrm{~ms})$ at the last couple of RT bins. The combined Simon effect function has a slightly positive slope, and not the negative slope customarily observed for left and right locations.

Wiegand \& Wascher (2001), Exp. 1

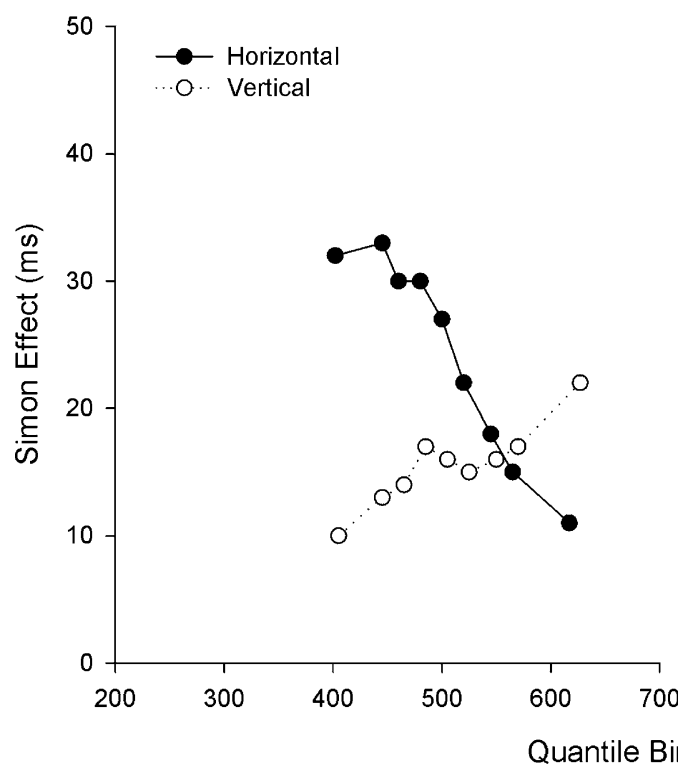

Fig. 5 The Simon effect magnitude (in milliseconds) for each bin as shown in Wiegand and Wascher's (2005) Experiment 1 (left panel) and Proctor et al.'s (2003) Experiment 1 (right panel), respectively. A decreasing function for horizontal stimuli (plotted by the bold line in both figures) and an increasing time course for vertically aligned ones, were obtained in both experiments. Left panel from Wiegand and Wascher (2005), Dynamic aspects of stimulus-response correspon-
Wiegand and Wascher (2005, Experiment 1) replicated the increasing Simon effect function for the vertical dimension and the typical decreasing effect function for the horizontal dimension in a single experiment (Fig. 5, left panel). As in Wascher et al. (2001), participants responded to the letter $A$ or $B$, this time presented in one of five boxes, one at fixation and the others to the left, right, top, and bottom of the fixated box (with the remaining boxes filled with neutral filler lines). The response keys were arrayed vertically, and half of the participants responded with the right hand on the top key and the left on the bottom, and vice versa for the other half. The Simon effect for vertical stimulus location with key location increased from $10 \mathrm{~ms}$ at the shortest of nine RT bins to $23 \mathrm{~ms}$ at the longest. In contrast, the Simon effect for left and right stimulus locations with left and right hands showed the typical decreasing function (beginning at 30-35 ms and decreasing to about $10 \mathrm{~ms}$ ). This result pattern is consistent with the distinction between the translation mechanism and visuomotor activation, because the above-below stimuli did not have an anatomical correspondence with the left and right hands, whereas the left-right stimuli did, which would lead to increasing and decreasing functions, respectively.

Proctor, Vu, and Nicoletti (2003) examined the Simon task for a two-dimensional version of the Simon task in which a single stimulus can correspond or not with the response on both the vertical and horizontal dimensions. In
Proctor et al. (2003), Exp. 1

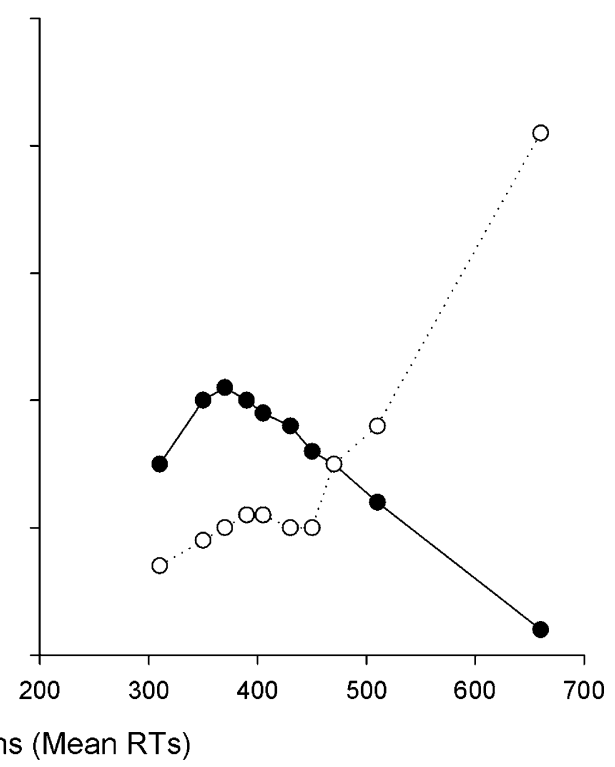

dence. Evidence for two mechanisms involved in the Simon effect. Journal of Experimental Psychology: Human Perception and Performance, 31, 453-464. Reprinted with permission of the American Psychological Association. Right panel from Proctor et al. (2003), Does right-left prevalence occur for the Simon effect? Perception \& Psychophysics, 65, 1318-1329. Reprinted with permission of the Psychonomic Society, Inc 
Experiment 1, a red or green stimulus was presented at one of four corners of an imaginary square. Responses were made with thumb presses of switches attached to one of two hand grips, one to the left and one to the right, mounted at different heights. With this arrangement, the two alternative responses were up-right paired with down-left or up-left paired with down-right, and a stimulus could correspond on one dimension, both dimensions, or neither. For example, with up-right/down-left responses, if the stimulus designated the up-right response button as correct, the correspondence relations of the stimulus locations would be as follows: The up-right location corresponds on both dimensions; the up-left location corresponds on the vertical dimension (and not on the horizontal dimension); the down-right location corresponds on the horizontal dimension (and not on the vertical dimension); and the down-left location corresponds on neither dimension. The Simon effect for correspondence on the horizontal dimension increased across the first 3 of 10 bins to a maximum of about $25 \mathrm{~ms}$ and then decreased to close to $0 \mathrm{~ms}$ over the remaining bins. In contrast, the Simon effect for correspondence on the vertical dimension increased over the first 9 bins from about 8 to $16 \mathrm{~ms}$ and was largest $(40 \mathrm{~ms})$ at the last RT bin (Fig. 5, right panel).

Wiegand and Wascher's (2005) Experiment 2 was similar to Proctor et al.'s (2003) Experiment 1, but requiring a response to the letter $A$ or $B$ : Four possible boxes in which the stimulus could appear on any given trial were in a $2 \times 2$ array, as were the response keys, and the two keys on the positive or negative diagonal were pressed by the index fingers of each hand. Like Proctor et al.'s (2003) results, the vertical dimension showed an increasing Simon effect function, and the horizontal dimension a decreasing function, with the overall function for compatibilities on both dimensions being equal to the sums of their individual effects. The results of this experiment and those in Proctor et al. (2003) suggest that the processes producing the vertical and horizontal Simon effects operate together on a single trial and are independent of each other.

Proctor et al. (2003) conducted a second experiment in which the relative salience of the vertical and horizontal dimensions was varied by positioning the stimuli closer on one dimension (the less salient one) than on the other (the more salient one). As would be expected from such a manipulation of relative salience, the Simon effect increased (relative to an equally spaced neutral condition) for the more salient dimension and decreased for the less salient one. Critically, this factor did not interact with RT bin: The vertical Simon effect tended to increase across bins, and the horizontal Simon effect tended to decrease, regardless of the overall magnitude of the Simon effect for the condition. This result suggests that the temporal properties of activation for the two dimensions do not interact and remain the same despite differences in the size of the overall Simon effect.

Decreasing functions Although all of the studies above showed an increasing function for the time course of the vertical Simon effect, studies in which the S-R mappings varied from trial to trial have shown decreasing functions. For example, for stimuli presented in upper and lower locations, De Jong et al. (1994, Experiment 4) assigned a color-response mapping to top and bottom response keys and then presented a cue (SAME or OPPO) 1,200 ms prior to onset of the imperative stimulus to indicate whether to respond with the key assigned to the color or with the opposite key. On the compatible color-response mapping trials, the Simon effect decreased from $60 \mathrm{~ms}$ at the first of five RT bins to $30 \mathrm{~ms}$ at the last (see Valle-Inclán \& Redondo, 1998, for a similar result). Because of this discrepancy in results, Wiegand and Wascher (2007b) conducted experiments in which they varied whether the mappings were constant or varied randomly within trial blocks. Only vertical stimulus locations (designated by two boxes) were used, and responses were made with vertically arranged response keys, as in their 2005 Experiment 1. For the random mapping, the lowercase letters $a$ and $b$ appeared in the boxes for $200 \mathrm{~ms}$, and the target letter, an uppercase $A$ or $B$, appeared $400 \mathrm{~ms}$ after their offset; the correct response was the one corresponding to the box in which that letter had been cued. With the fixed mapping, the RT distribution was a slightly increasing function (the Simon effect was smaller at the shortest bin than at the others). However, with the random mapping, the effect decreased from about 35 to about $7 \mathrm{~ms}$.

Wiegand and Wascher's (2007b) Experiment 2 was similar, except that the cue was a centered circle or square, and the results were like those of Experiment 1. A comparable manipulation was used with horizontal stimulus and response locations in Experiment 3, and the function for the random mapping condition looked similar to that for the random vertical conditions in Experiments 1 and 2. To account for the finding that vertical orientations yield decreasing Simon effect functions when the relevant mapping varies randomly, Wiegand and Wascher (2007b) concluded, "Thus, for vertical S-R relations the alternation of the mapping rules seems to change the processing of spatial parameters in such a way that a visuomotor mechanism - that is, the activation of the corresponding response-is enabled" (p. 227). The authors speculated that "the varying mapping rules result in a stronger association between vertical response locations and the to-be-used hand" (p. 228). Such ad hoc statements were necessary because, according to Wascher et al.'s (2001) original two-process model, decreasing Simon effect functions for vertical S-R orientations should never be obtained, because there is no 
direct correspondence of the stimulus locations with the anatomical distinction between left and right hands.

Unimanual response sets and reaching movements

Another implication of Wascher et al.'s (2001) hypothesis is that visuomotor activation depends on correspondence between stimulus position and left or right anatomical status of the effectors because such activation occurs when each stimulus and corresponding response can be processed within the same cerebral hemisphere. Such activation should not occur when the responses are made with two fingers on a single hand or by movements of a single hand, because the same hemisphere controls all responses. To test this prediction, Wiegand and Wascher (2007a, Experiment 1) conducted an experiment similar to that in Wascher et al.'s study, but participants responded with the index and middle fingers of a single hand. For trial blocks in which the mapping of letters to responses was fixed as $A \rightarrow$ left and $B \rightarrow$ right, the Simon effect increased from $8 \mathrm{~ms}$ in the first of nine RT bins to about $25 \mathrm{~ms}$ in the fourth bin and then remained at approximately that value for the rest of the distribution. However, when the mapping of letters to keys varied randomly from trial to trial, being signaled by a centered form that appeared $600 \mathrm{~ms}$ before the target stimulus, the Simon effect started above $20 \mathrm{~ms}$ at the first bin, peaked at about $27 \mathrm{~ms}$ in the third bin, and then decreased to slightly negative at the last bin.

Wiegand and Wascher (2007a) interpreted this latter finding as indicating that visuomotor activation is due not to spatioanatomical mapping, but to distinct motor codes: "A direct activation of the corresponding response seems to occur when there is in an overlap between the spatial stimulus feature and parameters of the motor code" (p. 409). However, because RT was longer in the random mapping condition, an alternative explanation is that the same activation/dissipation process was involved for both blocked and random presentation, with responses in the former condition occurring before the dissipation phase had been reached.

Consistent with the view that decreasing effect functions are not due to spatioanatomical mapping, in a study using color as the relevant dimension (without a noise stimulus opposite the target location), Proctor and $\mathrm{Vu}$ (2010) found the decreasing Simon effect function when the keypresses were made with the index and middle fingers of the same hand. In Experiment 1B, the Simon effect increased from 15 to $35 \mathrm{~ms}$ across the first 3 of $10 \mathrm{RT}$ bins but then decreased to about $-15 \mathrm{~ms}$ at the 10 th bin. Cho and Proctor (2010) obtained a similar pattern of decreasing Simon effect as RT increased, using within-hand keypresses for the color Simon task.
Wiegand and Wascher's (2007a) Experiment 2 was the same as their Experiment 1, except that left and right response buttons were operated by means of a single handle, held with the right hand, which had to be moved clockwise or counterclockwise for key operation. The results of this experiment showed even more clearly than those of Experiment 1 that, in the range where the RT distributions for the consistent and random letter-mapping conditions overlapped, the Simon effect function for the consistent mapping was similar to that for the random mapping. Wiegand and Wascher (2007a) concluded that the results were consistent with the idea that direct activation of the corresponding response occurs when the spatial stimulus feature and parameters of the motor code overlap. Rubichi and Pellicano (2004, Experiment 1) delayed responding by using a no-go stimulus (blue color) on $20 \%$ of the trials for a task in which participants responded to the red/green color of a stimulus (presented in one of three boxes located to the left of fixation in one trial block and to the right in another) with unimanual left-right aimed movements from a centralized button to lateralized response pads. Of most relevance, the Simon effect decreased monotonically from $36 \mathrm{~ms}$ in the first of five RT bins to $-25 \mathrm{~ms}$ in the last bin.

A final study to mention is that of Buetti and Kerzel (2008). They directly compared conditions in which responses were made by lifting the left or right index finger (i.e., bimanual response set) or by moving the right index finger from a fixation cross on a screen to one of two boxes (i.e., unimanual response set) that marked the locations at which a red or green stimulus could occur. Horizontal visual stimulus and response locations showed the typical decreasing Simon effect function, whereas vertical visual stimulus and response locations and horizontal auditory stimulus and response locations showed flat and slightly increasing functions, respectively. Of most importance, in all cases, there was no interaction of the RT distribution function with bimanual or unimanual response set, indicating that the RT distributions were similar across response sets.

\section{Summary}

The result patterns obtained with different variations of the Simon effect for physical location stimuli are generally consistent. The Simon effect peaks early in the RT distribution when the stimuli appear in left and right locations and there is a simple left- and right-response distinction. However, this decreasing function is not restricted to situations in which the stimulus and response alternatives have a correspondence of processing hemispheres, as Wascher et al.'s (2001) account proposed. It occurs for Simon effects obtained with the index and 
middle fingers of a single hand and with left and right movements of a single hand. In the former case, there are still left and right effectors, even though they are controlled by the same hemisphere, whereas in the latter case, there is not even an effector difference. Although vertical stimulus and response orientations typically show increasing Simon effect functions, the fact that the functions are of the decreasing type when the relevant S-R mapping is varied is also difficult to accommodate within Wascher et al.'s original visuomotor activation account. Both of these unexpected patterns of results led Wiegand and Wascher (2007a, 2007b) to offer ad hoc modifications of the account that have yet to be confirmed. Although visual anatomical mapping does not seem to be the critical factor, it remains possible that the decreasing Simon effect function is associated with some aspect of visual processing, since no variant of the auditory Simon effect has been reported, of which we are aware, that shows a decreasing function.

\section{The time course of Simon effects without physical locations}

Location information conveyed by properties of centrally presented objects and by location words (i.e., left/right) or symbols (i.e., arrows) has dimensional overlap on a conceptual level with keypress responses (Kornblum et al., 1990). Therefore, Simon effects for correspondence of this conceptual location information with left-right responses are obtained for these stimulus sets. Because of the emphasis that Wascher et al.'s (2001) account places on the horizontal S-R mapping for visuomotor activation to occur, it suggests that, even when responses are made with the left and right hands in a normal position, these other Simon effects (with the possible exception of the objectbased effect) should not show decreasing RT distribution functions. In the following, we examine the Simon effects for stimulus properties other than physical location to determine whether they show increasing or decreasing distribution functions. Major studies are summarized in Table 2.

\section{Object-based Simon effects}

A variant of the Simon effect that has received considerable interest is one in which the graspable handle of a visual object occurs to the left or right side. Tucker and Ellis (1998) presented participants with photographs of graspable objects for which the handle was lateralized to the left or right. The task was to classify the object as upright or inverted by pressing a left or right response key. A Simon effect based on the handle side (i.e., a benefit for correspondence of handle side and response location) was obtained when the task was performed with the index fingers of the two hands, but not when it was performed with the index and middle fingers of the right hand. Consequently, Tucker and Ellis (1998) concluded that "the results ... are consistent with the view that seen objects automatically potentiate components of the actions they afford" (p. 830).

Although Tucker and Ellis (1998) did not include RT distribution analyses of their data, subsequent studies of the object-based Simon effect have done so. Riggio et al. (2008) reported a distribution analysis for an experiment in which left and right keypresses were made with the index fingers to the upright-inverted orientations of kitchen objects with graspable handles. The object-based Simon effect increased from $7.5 \mathrm{~ms}$ at the two shortest RT quintiles to $17.5 \mathrm{~ms}$ at the two longest ones. This result was replicated by Iani, Baroni, Pellicano, and Nicoletti (in press), who used the same task as Riggio et al. (2008), with two main exceptions: The stimuli were presented laterally, and participants responded by performing reaching movements with the left or right hand. At the beginning of each trial, participants pressed left and right start keys with the index fingers. When the target appeared, they had to release, according to its vertical orientation (i.e., upward/ inverted), one of those start keys and reach to press the spatially corresponding response key, which could be a response box (Experiment 1) or a rectangular goal area on a touch screen device (Experiment 2). In Experiment 1, the object-based Simon effect was $9 \mathrm{~ms}$ overall. Its time course turned out to increase from 3 to $28 \mathrm{~ms}$ across five bins when the stimuli remained in view until the finger was lifted from the start key. When targets stayed on the screen until the response box was pressed, the effect remained rather constant (from 8 to $2 \mathrm{~ms}$ ). In Experiment 2, the object-based Simon effect showed a positive function only in combination with noncorresponding spatial Simon effect trials. In other words, the object-based effect reached significance and displayed an increasing effect function (from 8 to $41 \mathrm{~ms}$ ) just when the target position mismatched with the hand effect (e.g., when the stimulus was presented at the right of the screen and a left response had to be made).

Two main conclusions can be drawn from Iani et al.'s (in press) results. First, the object-based Simon effect showed an increasing time course, in most conditions reaching significance only at the later RT bins, probably because the effect requires semantic processing of the object (see also Symes, Ellis, \& Tucker, 2005; Vainio, Ellis, \& Tucker, 2007). Second, the object-based Simon effect arose, as does the spatial Simon effect, at a processing stage before response execution. This is shown by the significant interaction in Experiment 2: Only when the stimulus position did not match with the hand did the handle-hand 
Table 2 Overview of the most important parameters relating to the Simon effect (SE) for nonphysical spatial information and its time course across studies discussed in this article

\begin{tabular}{|c|c|c|c|c|c|c|c|}
\hline Stimulus & Response & Study & SE (ms) & SE Time Course & $\begin{array}{l}\text { SE Range } \\
\text { (ms) }\end{array}$ & $\begin{array}{l}\text { No. of } \\
\text { Bins }\end{array}$ & $\begin{array}{l}\text { RT Range } \\
\text { (ms) }\end{array}$ \\
\hline \multicolumn{8}{|l|}{ Object-Based Simon Effects } \\
\hline \multirow[t]{6}{*}{ Tool handles } & \multirow{6}{*}{$\begin{array}{l}\text { Horizontal left/right } \\
\text { hands }\end{array}$} & Cho and Proctor (2010) & & & & & \\
\hline & & Exp. 3 & 47 & increasing & $37-60$ & 4 & $410-730^{*}$ \\
\hline & & Iani et al. (in press) & & & & & \\
\hline & & Exp. 1 & 6 & stable & $9-2$ & 5 & N/A \\
\hline & & Riggio et al. (2008) & & & & & \\
\hline & & Exp. 1 (onset condition) & 19 & increasing & $8-20$ & 5 & $428-717$ \\
\hline \multicolumn{8}{|c|}{ Symbol- and Word-Based Simon Effects } \\
\hline \multirow[t]{4}{*}{ Arrows /arrow like stimuli } & \multirow{4}{*}{$\begin{array}{l}\text { Horizontal left/right } \\
\text { hands }\end{array}$} & Pellicano et al. (2009) & & & & & \\
\hline & & Exp. 1 & 19 & increasing then stable & $10-27$ & 5 & $250-400 *$ \\
\hline & & Proctor et al. (2009) & & & & & \\
\hline & & Exp. 1 & 44 & increasing then decreasing & $38-55$ & 4 & $350-620^{*}$ \\
\hline \multirow[t]{6}{*}{ Spatial words } & \multirow{6}{*}{$\begin{array}{l}\text { Horizontal left/right } \\
\text { hands }\end{array}$} & Miles and Proctor (2009) & & & & & \\
\hline & & Exp. 1 & 31 & increasing & $12-61$ & 4 & $370-570 *$ \\
\hline & & Pellicano et al. (2009) & & & & & \\
\hline & & Exp. 3 & 22 & increasing & $4-54$ & 5 & $320-600 *$ \\
\hline & & Proctor et al. (2009) & & & & & \\
\hline & & Exp. 1 & 28 & increasing & $0-70$ & 4 & $370-700 *$ \\
\hline \multicolumn{8}{|l|}{ SNARC Effects } \\
\hline \multirow[t]{6}{*}{$\begin{array}{l}\text { Number magnitude } \\
\text { (Parity Judgements) }\end{array}$} & \multirow[t]{6}{*}{$\begin{array}{l}\text { Horizontal left/right } \\
\text { hands }\end{array}$} & \multicolumn{6}{|l|}{$\begin{array}{l}\text { Gevers, Caessens, and Fias } \\
(2005)\end{array}$} \\
\hline & & Exp. 1 & 9 & stable & N/A & 5 & $355-635^{*}$ \\
\hline & & Gevers et al. (2006) & & & & & \\
\hline & & Exp. 1 & 9 & increasing & $\mathrm{N} / \mathrm{A}$ & 4 & N/A \\
\hline & & Mapelli et al. (2003) & & & & & \\
\hline & & Exp. 1 & 12 & stable & N/A & 5 & N/A \\
\hline
\end{tabular}

Note. Values designated with asterisks are approximated values

correspondence emerge. The lack of this interaction in Experiment 1 was due, according to the authors, to the less ecological nature of the response movements in that experiment. Whereas in Experiment 1 the reaching movements were directed toward two external response boxes, in Experiment 2 participants acted directly on the screen, closer to the stimuli and, thus, to the spatial information conveyed by their handles.

Cho and Proctor (2010) also used graspable objects (frying pans), to which responses were made according to the objects' upright/inverted orientation or color. Their results showed an increasing Simon effect, and this pattern was evident both when the two fingers making the keypresses were on the same hand and when they were on different hands. Cho and Proctor (in press) extended their findings to teapot stimuli, finding that regardless of whether the teapots were complete or included only a handle or spout, the Simon effect was smallest at the shortest of four RT bins and increased across the distribution. That similar distribution functions are obtained within and between hands with object stimuli is counter to the explanation in terms of an affordance for grasping with the left or right hand. Moreover, the positive slope of these functions implies that the object-based Simon effect is not due to direct visuomotor activation of the type proposed by Wascher et al. (2001), although they attributed such activation to the grasping system.

Increasing functions for the object-based Simon effect were also found when participants made a power or precision grip response to natural versus manufactured objects (Tucker \& Ellis, 2001). Responses were faster when the grip response corresponded with that appropriate for the object, and this effect was smallest for the first of five RT bins and then increased linearly across the bins. The one 
exception was an experiment in which the object did not remain on the screen - in which case, the function peaked at the middle bin and then decreased. Derbyshire, Ellis, and Tucker (2006) obtained similar increasing object-based Simon effect functions for responses to an object presented in a multiobject array and cued by an arrow. One interpretation of these results is that the time course of activation is longer for objects with a left versus right feature distinction (e.g., handle) than for stimuli occurring at left or right physical locations.

Symbol- and word-based Simon effects

Location information can be conveyed by left- versus rightpointing arrows and the words left or right. These stimuli produce activation of the corresponding keypress response that would be expected to dissipate if dissipation were a general property of automatic activation of left and right responses. However, studies have shown that this does not occur. Pellicano, Lugli, Baroni, and Nicoletti (2009) conducted two experiments in which participants responded to the color of centered arrows, with and without a deadline of $550 \mathrm{~ms}$. The Simon effect was $10-15 \mathrm{~ms}$ at the first of five RT bins and increased to about $30 \mathrm{~ms}$ at the last two bins, tending to level off. Although the pattern was not as strong, Proctor et al. (2009) found, in their control condition for which people performed only the arrows Simon task, an increase from about 38 to $55 \mathrm{~ms}$ across the first three of four RT bins, with a return to $38 \mathrm{~ms}$ in the final bin. These studies provide no evidence that the Simon effect for arrow direction decreases as RT increases.

Pellicano et al.'s (2009) Experiment 3 used centered location words, and the Simon effect increased from less than $10 \mathrm{~ms}$ at the two shortest RT bins to $55 \mathrm{~ms}$ at the longest bin. Proctor et al. (2009, Experiment 1) likewise found the Simon effect for location words to increase from 2 to $70 \mathrm{~ms}$ across four RT bins, and Miles and Proctor (2009, Experiment 1) obtained a similar result for their condition with intact words $(12 \mathrm{~ms}$ at the first to $61 \mathrm{~ms}$ at the last of four bins).

\section{SNARC effects}

Spatial associations with more abstract symbolic information also yield Simon-type effects. The most widely investigated of these effects is the spatial-numerical association of response codes (SNARC) effect, in which the relative magnitude of presented numbers influences left-right responses, with low and high numbers biasing left and right responses, respectively (Dehaene, Bossini, \& Giraux, 1993). The SNARC effect occurs regardless of the task relevance of number magnitude, which leads to an S-R correspondence effect resembling those found in other Simon tasks, especially word- and arrow-based effects that also do not rely on their location in space but, rather, on spatial information extracted from their meaning.

Similar to the arrow-based Simon effect, there is evidence that the SNARC effect shows a relatively stable correspondence effect across all RT bins. For example, Mapelli, Rusconi, and Umiltà (2003) had participants make odd-even parity judgments to numbers between 1 and 9 (excluding 5), presented on the left or right side of a display. In Experiment 1, a standard location-based Simon effect was obtained that was largest at the shortest of five RT bins and then declined, being absent at the longest bin. In contrast, the SNARC effect was stable across RT bins and was additive with the effect of location. Mapelli et al. decreased stimulus size and increased stimulus distance from fixation in Experiment 2 to lengthen RT. In this case, the location-based Simon effect became negative by the longest bin. However, the SNARC effect still remained unchanged across RTs. Because the Simon effect decreased across RT bins but the SNARC effect did not, the authors argued that these effects are due to different mechanisms.

Subsequently, Gevers, Verguts, Reynvoet, Caessens, and Fias (2006) had participants perform the more standard SNARC task in which the parity judgments are made to centrally presented numbers. The SNARC effect gradually increased from the shortest of four RT bins to the longest. Because Gevers et al.'s (2006) version of the SNARC task was more pure than that of Mapelli et al. (2003), it is reasonable to conclude that the effect of number magnitude on the correspondence effect tends to increase as RTs get longer.

\section{Can the effect functions be attributed to the time course of response activation?}

To this point, we have interpreted the Simon effect functions in terms of the time course of activation. Given Zhang and Kornblum's (1997) argument that the differences in variability producing the functions could arise in other ways, it is important to evaluate whether converging evidence with other temporal manipulations indicates that the distribution functions indeed reflect mainly temporal factors. Several results provide such converging evidence.

Converging behavioral evidence

Standard left-right Simon effect For the left-right Simon effect with keypresses, one corroborating finding already mentioned is that of Simon et al. (1976), showing that the visual Simon effect was eliminated when the response had 
to be withheld until a spatially neutral go signal occurred. In more detail, in Simon et al.'s Experiment 1, participants performed the standard color version of the Simon task, with the major alteration being that a tone occurred as a go signal to respond after a stimulus onset asynchrony (SOA) of $0,150,250$, or $350 \mathrm{~ms}$, randomly intermixed. A Simon effect of 35-40 ms was evident at the 0-ms SOA, for which there was no response delay, and the effect decreased systematically as SOA increased, being absent at the longest SOA.

Hommel (1993) found that several manipulations that slowed coding of the relevant stimulus information, relative to that of the irrelevant stimulus location, decreased the Simon effect. The effect was reduced by increasing stimulus eccentricity, reducing the quality of the relevant stimulus dimension with overlaid patterns, and decreasing contrast of the stimulus to the background. In Hommel's (1993) last experiment, the visual stimuli were built up over time, with the complete stimulus not being available until $196 \mathrm{~ms}$ after initial onset. The Simon effect for this condition was smaller than that for a condition in which the entire stimulus was available immediately at onset (see the earlier discussion of Hommel, 1994b, using this manipulation).

Hommel (1994a) obtained similar results for a situation in which the imperative stimulus appeared in one of two boxes, both positioned to the left or right of fixation. A Simon effect with regard to the two boxes was obtained for color judgments, but not for square/rectangle judgments, which were more difficult and showed longer overall RTs. Further corroborating evidence is provided by Roswarski and Proctor's (1996) Experiments 1 and 3, in which a stimulus could occur in one of eight or four positions, respectively. In their study, for the leftmost and rightmost stimulus positions, in which the stimuli were left or right according to all frames of reference, RT was shorter for color judgments than for circle/square judgments, which, in turn, was shorter than for rectangle/square judgments. In turn, the Simon effect was largest for the color judgments, intermediate for circle/square judgments, and smallest for rectangle/square judgments. Rubichi, Nicoletti, Iani, and Umiltà (1997) obtained similar results using shape judgments for easy and difficult tasks. In their Experiments 1 and 2, a go/no-go signal was presented above fixation before appearance of a stimulus for $100 \mathrm{~ms}$, to which a shape judgment (i.e., rectangle/square) was to be made by pressing a left/right key. When the rectangle differed sufficiently from the square to make the discrimination easy (Experiment 2), the Simon effect was $18 \mathrm{~ms}$, decreasing from $41 \mathrm{~ms}$ at the first of five RT bins to $-16 \mathrm{~ms}$ at the last bin. With a more difficult discrimination (Experiment 1), the Simon effect reversed to $-22 \mathrm{~ms}$ overall, changing from $-6 \mathrm{~ms}$ in the first bin to $-27 \mathrm{~ms}$ in the last one.
That the mean Simon effect is smaller when responding is delayed is consistent with the hypothesis that activation of the corresponding response is at a maximum shortly after stimulus onset and then decreases, as proposed by Hommel (1993) in his temporal overlap account and De Jong et al. (1994) in their two-process model. The Simon effect is strongest during the period in which the irrelevant activation of the corresponding response overlaps with that of activation of the task-defined response. This hypothesis does not predict the inversion found by Rubichi et al. (1997) in their Experiment 1, unless it is assumed that there is subsequent inhibition of the initial spatial code or activation of an opposing spatial code. Rubichi et al. (1997) interpreted the reversal of the Simon effect according to an attention shift account (see Nicoletti \& Umiltà, 1994; Stoffer, 1991). In Experiment 1, the first shift of attention caused by the abrupt onset of the stimulus would be followed by a second one back to fixation. Because the form discrimination was difficult, the relevant stimulus code would become available relatively late in processing, when attention was being shifted toward fixation, where both the feedback and the next trial were to appear. In Experiment 2, because the form discrimination was easy, most of the responses would be emitted prior to the second attention shift toward fixation

$\mathrm{Lu}$ and Proctor (1994) examined several task variations, including whether the relevant stimulus dimension was nonspatial, as in the typical Simon task, or spatial (left- and right-pointing arrow direction), as in what is sometimes called the spatial Stroop task. Factors that slowed responding to the relevant stimulus dimension resulted not only in smaller Simon effects with compatible mappings of the relevant information to responses, but also in larger negative Simon effects (i.e., Hedge and Marsh reversals) with incompatible mappings. The latter result matches the increasing size of the negative Simon effect evident in the distribution functions with incompatible color mappings (De Jong et al., 1994).

Finally, Burle, van den Wildenberg, and Ridderinkhof (2005) combined an SOA manipulation with an RT distribution analysis to evaluate whether these two techniques are sensitive to the same cognitive mechanism. They conducted two experiments in which a lateral cue could precede (negative SOA) or follow (positive SOA) the imperative stimulus (bilateral colored shape), to which a left/right response was to be made, allowing measurement of mean RT and the distribution functions at the different SOAs (ranging from -400 to $+300 \mathrm{~ms}$ in Experiment 1 and from -150 to $+150 \mathrm{~ms}$ in Experiment 2). Results showed that the size of the mean Simon effect and the slopes of the distribution functions were positively correlated. At negative SOAs (where the lateralized cue preceded the bilateral target), the mean Simon effect was negative, and the size of 
this effect corresponded to a steeper slope for the negative effect function; the opposite was true for positive SOAs. The switch between the negative and positive effect patterns took place at 0 -ms SOA, the condition most similar to a normal Simon task. Burle et al. (2005) stated that "the effects of SOA manipulation and the results of the distributional analysis converged and were positively correlated, suggesting that they involve a common mechanism of response activation followed by the selective suppression of that activation" (p. 619).

Vertical versus horizontal Simon effects As has been mentioned, the first two experiments of Wiegand and Wascher (2005) compared vertical and horizontal versions of the Simon task to show that the RT distribution functions reflected temporal properties of response activation. Their Experiment 3 used speed stress instructions (respond faster than $500 \mathrm{~ms}$ ) to get participants to respond during the normally short RT end of the distributions. Under speed stress, the Simon effect was larger for the horizontal dimension than for the vertical dimension, consistent with the view that the horizontal activation occurs quickly. Experiment 4 used a dual-task, psychological refractory period method, with the Simon task being the second of two tasks performed on each trial. The intent was to slow responding in the Simon task at short SOAs. The vertical Simon effect was independent of SOA in this dual-task context, but the horizontal Simon effect was smaller at short than at long SOAs, suggesting that the delay in response selection at short SOAs allowed time for the activation to dissipate. Thus, as with the previously cited findings for the standard left-right Simon effect and the Hedge and Marsh reversal, the manipulations of mean RT corroborate a temporal activation interpretation of the distribution functions.

Vallesi and Umiltà (2009) also used SOA manipulations to investigate the processes underlying the horizontal and vertical Simon effects. Response execution to a red or green square was delayed until onset of a go signal (a white square at the same location) in their Experiment 1, whereas the relevant color square was preceded by the white square as a precue in Experiment 2. SOAs ranging from 0 to $600 \mathrm{~ms}$ were used in both experiments. The pattern of results for the vertical and the horizontal tasks turned out to be very similar: The Simon effect after a corresponding trial decreased when SOA manipulations were used, as compared with when they were not (neutral condition). This suggests that automatic response activation was the mechanism underlying performance in both tasks. However, for both the horizontal and vertical conditions of Experiment 2, the Simon effect did not show a steep decrease with increasing SOA, as it did for Experiment 1. The authors argued that the Experiment 2 results may have been due to a double shift of attention, instead of a shared cognitive process (see Rubichi et al., 1997). The abrupt onset of the target color square may have caused an additional attention shift, resulting in formation of a new spatial code that reactivated the spatially corresponding response.

To prevent the possibility of a new spatial code being formed, Vallesi and Umiltà (2009) conducted a third experiment in which identification of the relevant dimension was delayed by decreasing the discriminability of the relevant color feature, rather than through manipulation of SOA. The stimuli used were red-and-black and green-andblack checkerboards. For the high-color-discriminability condition all of the pixels in the square regions were colored, whereas in the low-color-discriminability condition, $90 \%$ of the pixels were replaced randomly with black and white pixels. Different from the previous two experiments, the stimuli were accompanied by a contralateral noise stimulus (a black-and-white checkerboard). The greater difficulty in identifying the stimulus color in the low-color-discriminability condition reduced the Simon effect in the horizontal task (from 29 to $9 \mathrm{~ms}$ ), but not in the vertical task, where the Simon effect was 29 and $35 \mathrm{~ms}$ for the high- and low-discriminability conditions, respectively. On the basis of these results, the authors concluded, in accordance with Wiegand and Wascher (2005), that two different mechanisms cause the horizontal and vertical Simon effects.

Object-based and location word Simon effect Evidence consistent with a time course interpretation has also been obtained for object-based Simon effects. Phillips and Ward (2002) presented a frying pan stimulus centrally as a precue; after an SOA of $0,400,800$, or $1,200 \mathrm{~ms}$, a target stimulus designating a left or right response was superimposed on the pan. With a normal uncrossed hand placement, a crossed hand placement, and foot responses, the Simon effects increased as SOA increased, showing little difference between the response conditions. These results are in agreement with the distribution analyses for object-based Simon effects.

Finally, results obtained with location words provide similar confirmatory evidence. Miles and Proctor (2009, Experiment 1) used a pattern mask that degraded discriminability of the irrelevant location word, but not of the relevant color in which the word was displayed. This manipulation eliminated the Simon effect at all bins, suggesting that activation of the response associated with the location word meaning was slowed enough that little activation occurred while participants were responding to color. In Experiment 2, the relevant stimulus dimension was changed to one of lower- versus uppercase letters for the location words, which were presented in black. In this case, the pattern mask can be assumed to disrupt 
processing of both the irrelevant location word feature and the relevant letter case feature. The nondegraded condition showed the Simon effect to increase across the RT distribution, as in the control condition of Experiment 1, described earlier, and the degraded condition showed an increasing function as well (shifted to longer RTs by more than $100 \mathrm{~ms}$ ). That the increasing function was unaffected when both the irrelevant and relevant dimensions were degraded is consistent with the view that the timing of activation of the irrelevant word information, relative to that produced by the relevant information, is crucial to the shape of the distribution function.

Summary The primary conclusion to draw from the studies described in this section is that, across a variety of types of Simon effect, time course and SOA manipulations produce results that coincide closely with those obtained from the distribution analyses. Therefore, the converging evidence from several manipulations indicates that the pattern of the Simon effect across the RT distribution reflects mainly the temporal properties of response activation, as De Jong et al. (1994), Ridderinkhof (2002a), and Wascher et al. (2001) assumed.

\section{Converging neurophysiological evidence}

Data from neurophysiological research further corroborate the view that changes in the Simon effect across the RT distribution indeed represent changes in response activation. De Jong et al. (1994) included an LRP measure in their Experiment 4, a technique that De Jong, Wierda, Mulder, and Mulder (1988) developed concurrently with Gratton et al. (1988). The LRP measures the difference between precentral motor cortex activation in the left and right hemispheres. Stimulus location and goaloriented response selection contribute to this activation, and thus corresponding trials in the Simon task will lead to greater activation on the side contralateral to the correct response than will noncorresponding trials. Although mapping properties of LRP activation to the timing of behavioral effects is not without problems, it seems that if stimulus-induced response activation declines along with the Simon effect across the RT distribution, this LRP difference should also be greatest soon after localization of the target stimulus (Wascher, 2005) and should disappear as time passes. De Jong et al.'s (1994) results confirmed this view; activation of the precentral motor cortex on the side contralateral to the correct response was greater for corresponding than for noncorresponding trials from approximately 100 to $300 \mathrm{~ms}$ after stimulus onset, but then activation of the correct response was roughly equivalent in both trial types.
Although, as was shown in De Jong et al. (1994), EEG data have the potential to provide insight into the nature of the Simon effect across RT distributions, there are several inconsistencies in the research that make this relationship less clear. In particular, if a declining Simon effect is reflected in declining LRP effects, it is reasonable to predict that an increasing Simon effect should also show increasing LRP differences over time. However, this does not appear to be the case. For example, Vallesi et al. (2005) found an increasing Simon effect across RTs for vertical stimuli, but, as in De Jong et al.'s (1994) study, the difference in activation of the parietal cortex contralateral to the correct response side was greater on corresponding than on noncorresponding trials early on (approximately 200$250 \mathrm{~ms}$ after stimulus onset) and then switched such that the noncorresponding trial more strongly activated the correct response. In the Simon task with horizontal stimuli and responses, the authors found that the Simon effect and LRP difference were greatest early in time and then both decreased. Vallesi et al. concluded that the inconsistency between the Simon effect and LRP measures in the vertical, but not the horizontal, Simon task points to separate mechanisms and that the Simon effect in the vertical task may not be closely related to the pattern of activation seen in LRPs.

Few functional magnetic resonance imaging (fMRI) studies have examined patterns of brain activation associated with the time course of the Simon effect-likely, because fMRI lacks the temporal precision for such investigations. However, Forstmann et al. (2008) examined brain activation associated with the pattern of changes in the Simon effect at different parts of the RT distribution. They argued that individual differences in the Simon effect can be attributed to both increases in visuomotor activation and then suppression of this activation, which rely on separate brain regions. They examined brain activation as a function of changes in the Simon effect over the RT distribution. The amount of suppression of visuomotor activation was operationalized as the slope of the declining Simon effect near the end of the RT distribution in the standard Simon task. Inferior frontal cortex (IFC) activation during the last portion of the RT distribution was strongly correlated with the negative slope of the Simon effect:- the more negative this slope, the greater the activation of IFC. IFC is generally regarded as an area that is pivotal in inhibitory processes (e.g., Aron et al., 2004), and therefore, this result nicely fits with the view that the declining Simon effect near the end of the RT distribution is associated with suppression of prepotent response activation. Importantly, IFC activation was not related to the pattern of the Simon effect during the early or middle segments of the RT distribution, in which the Simon effect often increases in magnitude or remains stable. 
In sum, results obtained using a variety of methodologies depict a coherent picture and one very similar to the one obtained with the RT distribution analysis, providing converging evidence that the distribution functions reflect primarily the time course of response activation.

\section{Conclusions}

Analyses of the Simon effect and related phenomena across the RT distribution have been used as a tool for analyzing the time course of information processing. Such analyses were first used by De Jong et al. (1994) to provide evidence for a two-process (conditional and unconditional) model of the Simon effect and the Hedge and Marsh reversal (Hedge \& Marsh, 1975), for which a decreasing function for the Simon effect (and increasing function for the reversal) was attributed to rapid unconditional activation of the corresponding response, followed by dissipation. Since that time, most authors have interpreted the results of distribution analyses in terms of the time course of activation of the corresponding response code, as De Jong et al. (1994) did. From the beginning, though, critics have questioned whether the distribution functions of Simon effects are indeed indicative of the time course of information processing (Buetti \& Kerzel, 2008; Roswarski \& Proctor, 2003; Zhang \& Kornblum, 1997). Most notably, Zhang and Kornblum showed that the changes in size of the Simon effect across RT bins "are mathematically derivable from the statistical properties of the RT distributions [the relative variability of those for corresponding and noncorresponding trials] and are not necessarily related to the time-course assumptions" (p. 1544).

One goal of the present article was to answer the question of whether or not the distribution functions for the Simon effect in fact reflect the time course of activation by examining evidence from other methods in which response time is varied more directly. The data we have reviewed, some of which are summarized in Tables 1 and 2, show remarkable convergence in providing a positive answer that, yes, for the most part, the distribution functions reflect the time course of processing. In general, for task variations that yield a decreasing function, manipulations that slow responding to the relevant information, increasing mean RT, reduce the overall Simon effect, whereas for task variations that yield an increasing function, such manipulations increase the size of the overall Simon effect. There are likely cases in which the distribution functions are influenced by other factors, but our examination of existing results indicates that, for the most part, one can be relatively sure that the changes in Simon effect across the RT distribution reflect temporal activation properties.
A second goal of the article was to determine the consistency of the distribution within and between various task variations. Here, again, the results are relatively clear and straightforward. The decreasing functions originally reported by De Jong et al. (1994) are widely replicable for variants of the standard version of the Simon task in which left and right responses are made to a stimulus feature other than location but the stimuli appear in left and right locations. Sometimes, there is an initial increase, followed by the decrease, resulting in a J-shaped function, but the decrease is reliably obtained. Somewhat surprisingly, though, the decreasing function has mainly been restricted to the standard Simon task (see Fig. 1). For versions in which the irrelevant information is conveyed by centrally presented spatial indicators, such as arrows or location words, or by graspable object parts (i.e., handles), it is not found. Nor is a decreasing function obtained for location versions of the Simon task in which the stimuli and responses differ along the vertical dimension, rather than the horizontal dimension, or in which left-right location is conveyed implicitly, as in the SNARC effect. These results have been obtained reliably as well.

The major empirical findings are summarized as follows:

1. As has been noted, the standard left-right version of the Simon task, for which responses are made with the left and right hands, consistently shows the Simon effect to decrease from the short RT bins (sometimes after an initial increase) to the long ones.

2. A decreasing Simon effect function is often also obtained when keypress responses are made with the index and middle fingers of one hand and when the responses are left-right movements of a single hand, indicating that the distinction between left and right hands is not necessary to obtain the decreasing function.

3. In the standard Simon task, the response activation tends to dissipate sometime around 300 and $400 \mathrm{~ms}$, as indicated by the behavioral studies (both SOA and RT distribution functions) and by the response-related cortical activation.

4. Instead of decreasing, the Simon effect increases as RT increases for the standard left-right auditory Simon task and, for both the auditory and visual task, when responses are made with crossed hands. The decreasing function thus is not solely a consequence of spatial correspondences and depends on stimulus modality and effector placement.

5. Versions of the visual Simon task for which the stimulus and response locations are arrayed vertically, rather than horizontally, also yield functions that tend to increase across the RT distribution. However, when, in these tasks, the mapping of the relevant stimulus 
dimension to responses is mixed and signaled by a cue, the Simon effect decreases across the RT distribution, yielding a function similar to the one obtained in the standard Simon task.

6. Increasing Simon effect functions are generally obtained in tasks for which the location information is provided not by way of explicit physical locations but by central spatial indicators (i.e., arrows or words), graspable object parts (i.e., handles), and implicit locations on a number line (i.e., the SNARC effect). Complex stimuli in such tasks may delay the rate of response activation from the irrelevant stimulus dimension and the following dissipation of that activation.

The two most prominent models accounting for when, where, and why the various Simon effect functions are obtained are those of Ridderinkhof (2002a, 2002b) and Wascher et al. (2001). Ridderinkhof's explanation attributes the decreasing function to suppression of the automatic activation. The implication is that decreases should not be observed for situations in which there is no reason to suppress the activation or for individuals who are impaired in their suppression ability. Results from his research are generally consistent with these implications, with perhaps the strongest evidence being that the Simon effect function often does not decrease as much (if any) across the RT distribution for persons who are presumed to have reduced suppression capabilities. For example, several studies on aging and on $\mathrm{AD}$ and PD patients (see Fig. 3) indicate that reduced control of interference leads to less dissipation. However, the account offers no explanation for the systematic relations between various stimulus and response properties that determine whether the function is decreasing or increasing.

Wascher et al.'s (2001) account is specific in attributing the decreasing function to intrahemispheric activation resulting from a privileged visual-motor pathway. Thus, decreasing Simon effect functions should be restricted to situations in which the stimuli are varying in left and right visual locations and the left and right hands are in a normal uncrossed placement. In all other cases, the Simon effect functions should increase as RT increases, reflecting interference between codes. The initial results obtained by Wascher et al. and Wiegand and Wascher (2005) tended to conform to these predictions, showing increasing functions when the hands were crossed, when the stimuli were auditory, and when the stimulus and response sets were arrayed vertically (see Figs. 4 and 5). However, their subsequent studies (Wiegand \& Wascher, 2007a, 2007b) obtained evidence inconsistent with the original hypothesis, including decreasing functions for keypresses made by fingers on the same hand and for unimanual movement responses, as well as decreasing functions for vertically arrayed stimulus and response sets in some circumstances. These discrepancies have necessitated modifications of the theory, which as yet have not received independent confirmation.

To summarize, although the evidence does indicate that the distribution functions for the Simon effect reflect the time course of activation of the corresponding response, the understanding of changes in the Simon effect across RT distributions is incomplete. Whether a decreasing function reflects active suppression, passive decay, or both is not settled, nor is there a theoretical framework that captures adequately the conditions under which decreasing or increasing effect functions will be obtained. In other words, although the patterns of these distribution functions for different versions of the Simon task are generally reliable, there is no current explanatory model that encompasses both when and why the Simon effect decreases in some cases and increases in others.

That the results for variants of the spatial Simon task are reliable and likely representative of the temporal properties of response activation implies that the same should be true for other tasks in which irrelevant stimulus information produces response competition (e.g., Roelofs, 2008; Steinhauser \& Hübner, 2009; Wylie et al., 2009). Systematic comparisons of RT distributions across tasks should reveal the factors that influence the time course of response activation and the generalizability of those factors. As an illustration, Pratte, Rouder, Morey, and Feng (2010) recently reported a study comparing RT distribution functions for Stroop and Simon tasks. When the colors of stimuli were responded to with left and right keypresses, the Simon effect for left-right physical locations decreased across the RT distribution, whereas the Stroop effect for correspondence of irrelevant color words with the stimulus colors increased. Pratte et al. also showed this difference in distribution functions for spatial Stroop tasks in which keypress responses were made to location words left and right presented in left or right locations. When word identity was relevant and physical location irrelevant, the Simon effect was a decreasing function of RT, but when physical location was relevant and word identity irrelevant, the Stroop effect was an increasing function of RT. Abovebelow versions of the tasks yielded similar results, except for the time course of the Simon effect, which turned from decreasing to constant. These results confirm that irrelevant words produce an increasing RT function in a range of task contexts, whereas irrelevant physical locations produce a decreasing function only under more specific conditions. Complete accounts of the distribution functions must be able to explain these and other reliable findings.

Author Note Preparation of this manuscript was supported in part by ARO MURI Grant W911NF-05-1-0153. Giulia Baroni was a visiting scholar at Purdue University during the writing of the manuscript. We thank Bernhard Hommel, Edmund Wascher, and an anonymous reviewer for helpful comments on an earlier draft. 


\section{References}

Aron, A. R., Robbins, T. W., \& Poldrack, R. A. (2004). Inhibition and the right inferior frontal cortex. Trends in Cognitive Sciences, 8 , 170-177. doi:10.1016/j.tics.2004.02.010

Band, G., Ridderinkhof, K., \& van der Molen, M. (2003). Speedaccuracy modulation in case of conflict: The roles of activation and inhibition. Psychological Research, 67, 266-279. doi:10.1007/s00426-002-0127-0

Buetti, S., \& Kerzel, D. (2008). Time course of the Simon effect in pointing movements for horizontal, vertical, and acoustic stimuli: Evidence for a common mechanism. Acta Psychologica, 129, $420-428$.

Buhlmann, I., Umiltà, C., \& Wascher, E. (2007). Response coding and visuomotor transformation in the Simon task: The role of action goals. Journal of Experimental Psychology: Human Perception and Performance, 33, 1269-1282. doi:10.1037/00961523.33.6.1269

Burle, B., Possamaï, C.-A., Vidal, F., Bonnet, M., \& Hasbroucq, T. (2002). Executive control in the Simon effect: An electromyographic and distributional analysis. Psychological Research, 66, 324-339. doi:10.1007/s00426-002-0105-6

Burle, B., van den Wildenberg, W. P. M., \& Ridderinkhof, K. R. (2005). Dynamics of facilitation and interference in cue-priming and Simon tasks. European Journal of Cognitive Psychology, 17, 619-641. doi:10.1080/09541440540000121

Castel, A. D., Balota, D. A., Hutchison, K. A., Logan, J. M., \& Yap, M. J. (2007). Spatial attention and response control in healthy younger and older adults and individuals with Alzheimer's disease: Evidence for disproportionate selection impairments in the Simon task. Neuropsychology, 21, 170-182. doi:10.1037/ 0894-4105.21.2.170

Cho, D. (T.), \& Proctor, R. W. (2010). The object-based Simon effect: Grasping affordance or relative location of the graspable part? Journal of Experimental Psychology: Human Perception, and Performance, 36, 853-861. doi:10.1037/a0019328

Cho, D. (T.), \& Proctor, R. W. (in press). Correspondence effects for objects with opposing left and right protrusions. Journal of Experimental Psychology: Human Perception, and Performance.

Davranche, K., \& McMorris, T. (2009). Specific effects of acute moderate exercise on cognitive control. Brain and Cognition, 69, 565-570. doi:10.1016/j.bandc.2008.12.001

Dehaene, S., Bossini, S., \& Giraux, P. (1993). The mental representation of parity and numerical magnitude. Journal of Experimental Psychology: General, 122, 371-396. doi:10.1037/00963445.122.3.371

De Jong, R., Liang, C.-C., \& Lauber, E. (1994). Conditional and unconditional automaticity: A dual-process model of effects of spatial stimulus-response correspondence. Journal of Experimental Psychology: Human Perception and Performance, 20, 731750. doi:10.1037/0096-1523.20.4.731

De Jong, R., Wierda, M., Mulder, G., \& Mulder, L. J. M. (1988). The use of partial information in response processing. Journal of Experimental Psychology: Human Perception and Performance, 14, 682-692. doi:10.1037/0096-1523.14.4.682

Derbyshire, N., Ellis, R., \& Tucker, M. (2006). The potentiation of two components of the reach-to-grasp action during object categorisation in visual memory. Acta Psychologica, 122, 7498. doi:10.1016/j.actpsy.2005.10.004

Forstmann, B., van den Wildenberg, W., \& Ridderinkhof, K. (2008). Neural mechanisms, temporal dynamics, and individual differences in interference control. Journal of Cognitive Neuroscience, 20, 1854-1865. doi:10.1162/jocn.2008.20122

Gevers, W., Caessens, B., \& Fias, W. (2005). Towards a common processing architecture underlying Simon and SNARC effects.
European Journal of Cognitive Psychology, 17, 659-673. doi:10.1080/09541440540000112

Gevers, W., Verguts, T., Reynvoet, B., Caessens, B., \& Fias, W. (2006). Numbers and space: A computational model of the SNARC effect. Journal of Experimental Psychology: Human Perception and Performance, 32, 32-44. doi:10.1037/00961523.32.1.32

Gratton, G., Coles, M. G. H., Sirevaag, E. J., Eriksen, C. W., \& Donchin, E. (1988). Pre- and poststimulus activation of response channels: A psychophysiological analysis. Journal of Experimental Psychology: Human Perception and Performance, 14, 331-344. doi:10.1037/0096-1523.14.3.331

Hedge, A., \& Marsh, N. W. A. (1975). The effect of irrelevant spatial correspondence on two-choice response-time. Acta Psychologica, 39, 427-439. doi:10.1016/0001-6918(75)90041-4

Hommel, B. (1993). The relationship between stimulus processing and response selection in the Simon task: Evidence for a temporal overlap. Psychological Research, 55, 280-290. doi:10.1007/ BF00419688

Hommel, B. (1994a). Effects of irrelevant spatial S-R compatibility depend on stimulus complexity. Psychological Research, 56, 179-184. doi:10.1007/BF00419705

Hommel, B. (1994b). Spontaneous decay of response-code activation. Psychological Research, 56, 261-268. doi:10.1007/ BF00419656.

Hommel, B., Proctor, R. W., \& Vu, K.-P. L. (2004). A feature-integration account of sequential effects in the Simon task. Psychological Research, 68, 1-17. doi:10.1007/s00426-003-0132-y

Iani, C., Baroni, G., Pellicano, A., \& Nicoletti, R. (in press). On the relationship between affordance and Simon effects: Are the effects really independent? Journal of Cognitive Psychology.

Juncos-Rabadán, O., Pereiro, A. X., \& Facal, D. (2008). Cognitive interference and aging: Insights from a spatial stimulus-response consistency task. Acta Psychologica, 127, 237-246. doi:10.1016/ j.actpsy.2007.05.003

Kornblum, S. (1994). The way irrelevant dimensions are processed depends on what they overlap with: The case of Stroop and Simon-like stimuli. Psychological Research, 56, 130-135. doi:10.1007/BF00419699

Kornblum, S., Hasbroucq, T., \& Osman, A. (1990). Dimensional overlap: Cognitive basis for stimulus-response compatibility-A model and taxonomy. Psychological Review, 97, 253-270. doi:10.1037/0033-295X.97.2.253

Kubo-Kawai, N., \& Kawai, N. (2010). Elimination of the enhanced Simon effect for older adults in a three-choice situation: Ageing and the Simon effect in a go/no-go Simon task. Quarterly Journal of Experimental Psychology, 63, 452-464. doi:10.1080/ 17470210902990829

Lu, C.-H., \& Proctor, R. W. (1994). Processing of an irrelevant location dimension as a function of the relevant stimulus dimension. Journal of Experimental Psychology: Human Perception and Performance, 20, 286-298. doi:10.1037/00961523.20.2.286

Lu, C.-H., \& Proctor, R. W. (1995). The influence of irrelevant location information on performance: A review of the Simon and spatial Stroop effects. Psychonomic Bulletin \& Review, 2, 174-207.

Mapelli, D., Rusconi, E., \& Umiltà, C. (2003). The SNARC effect: An instance of the Simon effect? Cognition, 88, B1-B10. doi:10.1016/S0010-0277(03)00042-8

Miles, J., \& Proctor, R. W. (2009). Reducing and restoring stimulusresponse compatibility effects by decreasing the discriminability of location words. Acta Psychologica, 130, 95-102. doi:10.1016/ j.actpsy.2008.10.005

Nicoletti, R., \& Umiltà, C. (1994). Attention shifts produce spatial stimulus codes. Psychological Research, 56, 144-150. doi:10.1007/BF00419701 
Pellicano, A., Lugli, L., Baroni, G., \& Nicoletti, R. (2009). The Simon effect with conventional signals: A time-course analysis. Experimental Psychology, 56, 219-227. doi:10.1027/16183169.56.4.219

Phillips, J. C., \& Ward, R. (2002). S-R correspondence effects of irrelevant visual affordance: Time course and specificity of response activation. Visual Cognition, 9, 540-548. doi:10.1080/ 13506280143000575

Pratte, M. S., Rouder, J. M., Morey, R. D., \& Feng, C. (2010). Exploring the differences in distributional properties between Stroop and Simon effects using delta plots. Attention, Perception, \& Psychophysics, 72, 2013-2025. doi:10.3758/APP.72.7.2013

Proctor, R. W., Pick, D. F., Vu, K.-P. L., \& Anderson, R. E. (2005). The enhanced Simon effect for older adults is reduced when the irrelevant location information is conveyed by an accessory stimulus. Acta Psychologica, 119, 21-40. doi:10.1016/j. actpsy.2004.10.014

Proctor, R. W., \& Shao, C. (2010). Does the contribution of stimulushand correspondence to the auditory Simon effect increase with practice? Experimental Brain Research, 204, 131-137. doi:10.1007/s00221-010-2284-5

Proctor, R. W., \& Vu, K.-P. L. (2010). Stimulus-response compatibility for mixed mappings and tasks with unique responses. Quarterly Journal of Experimental Psychology, 63, 320-340. doi:10.1080/17470210902925270

Proctor, R. W., Vu, K.-P. L., \& Nicoletti, R. (2003). Does right-left prevalence occur for the Simon effect? Perception \& Psychophysics, 65, 1318-1329.

Proctor, R. W., Yamaguchi, M., Zhang, Y., \& Vu, K.-P. L. (2009). Influence of visual stimulus mode on transfer of acquired spatial associations. Journal of Experimental Psychology: Learning, Memory, and Cognition, 35, 434-445. doi:10.1037/a0014529

Ratcliff, R. (1979). Group reaction time distributions and an analysis of distribution statistics. Psychological Bulletin, 86, 446-461. doi:10.1037/0033-2909.86.3.446

Ridderinkhof, K. R. (2002a). Activation and suppression in conflict tasks: Empirical clarification through distributional analyses. In W. Prinz \& B. Hommel (Eds.), Common mechanisms in perception and action: Attention and performance XIX (pp. 494-519). New York: Oxford University Press.

Ridderinkhof, K. R. (2002b). Micro- and macro-adjustments of task set: Activation and suppression in conflict tasks. Psychological Research, 66, 312-323. doi:10.1007/s00426-002-0104-7

Riggio, L., Gawryszewski, L. G., \& Umiltà, C. (1986). What is crossed in crossed-hand effects? Acta Psychologica, 62, 89-100. doi:10.1016/0001-6918(86)90006-5

Riggio, L., Iani, C., Gherri, E., Benatti, F., Rubichi, S., \& Nicoletti, R. (2008). The role of attention in the occurrence of the affordance effect. Acta Psychologica, 127, 449-458. doi:10.1016/j. actpsy.2007.08.008

Roelofs, A. (2008). Dynamics of the attentional control of word retrieval: Analyses of response time distributions. Journal of Experimental Psychology: General, 137, 303-323. doi:10.1037/ 0096-3445.137.2.303

Roswarski, T. E., \& Proctor, R. W. (2003). Intrahemispherical activation, visuomotor transmission, and the Simon effect: Comment on Wascher et al. (2001). Journal of Experimental Psychology: Human Perception and Performance, 29, 152-158. doi:10.1037/0096-1523.29.1.152

Roswarski, T. W., \& Proctor, R. W. (1996). Multiple spatial codes and temporal overlap in choice-reaction tasks. Psychological Research, 59, 196-211. doi:10.1007/BF00425834

Rubichi, S., Nicoletti, R., Iani, C., \& Umiltà, C. (1997). The Simon effect occurs relative to the direction of an attention shift. Journal of Experimental Psychology: Human Perception and Performance, 23, 1353-1364. doi:10.1037/0096-1523.23.5.1353
Rubichi, S., \& Pellicano, A. (2004). Does the Simon effect affect movement execution? European Journal of Cognitive Psychology, 16, 825-840. doi:10.1080/09541440340000367

Simon, J. R. (1990). The effects of an irrelevant directional cue on human information processing. In R. W. Proctor \& T. G. Reeve (Eds.), Stimulus-response compatibility: An integrated perspective (pp. 31-86). Amsterdam: North-Holland.

Simon, J. R., Acosta, E., Mewaldt, S. P., \& Speidel, C. R. (1976). The effect of an irrelevant directional cue on choice reaction time: Duration of the phenomenon and its relation to stages of processing. Perception \& Psychophysics, 19, 16-22.

Simon, J. R., \& Small, A. M., Jr. (1969). Processing auditory information: Interference from an irrelevant cue. Journal of Applied Psychology, 53, 433-435. doi:10.1037/h0028034

Steinhauser, M., \& Hübner, R. (2009). Distinguishing response conflict and task conflict in the Stroop task: Evidence from exGaussian distribution analysis. Journal of Experimental Psychology: Human Perception and Performance, 35, 1398-1412. doi:10.1037/a0016467

Stoffer, T. H. (1991). Attentional focussing and spatial stimulusresponse compatibility. Psychological Research, 53, 127-135. doi:10.1007/BF01371820

Stürmer, B., Leuthold, H., Soetens, E., Schröter, H., \& Sommer, W. (2002). Control over location-based response activation in the Simon task: Behavioral and electrophysiological evidence. Journal of Experimental Psychology: Human Perception and Performance, 28, 1345-1363. doi:10.1037/0096-1523.28.6.1345

Symes, E., Ellis, R., \& Tucker, M. (2005). Dissociating object-based and space-based affordances. Visual Cognition, 12, 1337-1361. doi:10.1080/13506280444000445

Thomas, E. A. C., \& Ross, B. H. (1980). On appropriate procedures for combining probability distributions within the same family. Journal of Mathematical Psychology, 21, 136-152. doi:10.1016/ 0022-2496(80)90003-6

Tucker, M., \& Ellis, R. (1998). On the relations between seen objects and components of potential actions. Journal of Experimental Psychology: Human Perception and Performance, 24, 830-846. doi:10.1037/0096-1523.24.3.830

Tucker, M., \& Ellis, R. (2001). The potentiation of grasp types during visual object categorization. Visual Cognition, 8, 769-800. doi: $10.1080 / 13506280042000144$

Vainio, L., Ellis, R., \& Tucker, M. (2007). The role of visuospatial attention in action priming. Quarterly Journal of Experimental Psychology, 60, 241-261. doi:10.1080/17470210600625149

Valle-Inclán, F., \& Redondo, M. (1998). On the automaticity of ipsilateral response activation in the Simon effect. Psychophysiology, 35, 366-371. doi:10.1017/S0048577298960917

Vallesi, A., Mapelli, D., Schiff, S., Amodio, P., \& Umiltà, C. (2005). Horizontal and vertical Simon effect: Different underlying mechanisms? Cognition, 96, B33-B43. doi:10.1016/j.cognition.2004.11.009

Vallesi, A., \& Umiltà, C. A. (2009). Decay of stimulus spatial code in horizontal and vertical Simon tasks. Journal of General Psychology, 136, 350-373. doi:10.1080/00221300903266580

Vincent, S. B. (1912). The function of the vibrissae in the behaviour of the white rat. Animal Behavior Monographs, 1(5).

Vu, K.-P. L., \& Proctor, R. W. (2008). Age differences in response selection for pure and mixed stimulus-response mappings and tasks. Acta Psychologica, 129, 49-60. doi:10.1016/j.actpsy.2008.04.006

Wallace, R. J. (1971). S-R compatibility and the idea of a response code. Journal of Experimental Psychology, 88, 354-360. doi: $10.1037 / \mathrm{h} 0030892$

Wascher, E. (2005). The timing of stimulus localisation and the Simon effect: An ERP study. Experimental Brain Research, 163, 430-439.

Wascher, E., Schatz, U., Kuder, T., \& Verleger, R. (2001). Validity and boundary conditions of automatic response activation in the 
Simon task. Journal of Experimental Psychology: Human Perception and Performance, 27, 731-751. doi:10.1037/00961523.27.3.731

Wiegand, K., \& Wascher, E. (2005). Dynamic aspects of stimulusresponse correspondence: Evidence for two mechanisms involved in the Simon effect. Journal of Experimental Psychology: Human Perception and Performance, 31, 453-464. doi:10.1037/ 0096-1523.31.3.453

Wiegand, K., \& Wascher, E. (2007a). Response coding in the Simon task. Psychological Research, 71, 401-410. doi:10.1007/s00426005-0027-1

Wiegand, K., \& Wascher, E. (2007b). The Simon effect for vertical S$\mathrm{R}$ relations: Changing the mechanism by randomly varying the S-R mapping rule? Psychological Research, 71, 219-233. doi:10.1007/s00426-005-0023-5
Wylie, S. A., Ridderinkhof, K. R., Bashore, T. R., \& van den Wildenberg, W. P. M. (2010). The effect of Parkinson's disease on the dynamics of on-line and proactive cognitive control during action selection. Journal of Cognitive Neuroscience, 22, 20582073. doi:10.1162/jocn.2009.21326

Wylie, S. A., van den Wildenberg, W. P. M., Ridderinkhof, K. R., Bashore, T. R., Powell, V. D., Manning, C. A., et al. (2009). The effect of Parkinson's disease on interference control during action selection. Neuropsychologia, 47, 145-157. doi:10.1016/j.neuropsychologia.2008.08.001

Zhang, J., \& Kornblum, S. (1997). Distributional analysis and De Jong, Liang, and Lauber's (1994) dual-process model of the Simon effect. Journal of Experimental Psychology: Human Perception and Performance, 23, 1543-1551. doi:10.1037/ 0096-1523.23.5.1543 